\title{
Exotic magnetic response of superconducting wires subject to synchronous and asynchronous oscillating excitations
}

\author{
H. S. Ruiz ${ }^{1, a)}$ and A. Badía-Majós ${ }^{2}$ \\ ${ }^{1}$ Materials Science Institute of Aragón (ICMA-CSIC), Universidad de Zaragoza, María de Luna 3, \\ E-50018 Zaragoza, Spain and Center for Advanced Power Systems, Florida State University, \\ Tallahassee, Florida 32310, USA \\ ${ }^{2}$ Departamento de Física de la Materia Condensada-ICMA, Universidad de Zaragoza-CSIC, \\ María de Luna 3, E-50018 Zaragoza, Spain
}

(Received 14 January 2013; accepted 3 April 2013; published online 21 May 2013)

\begin{abstract}
The time-dependent local electromagnetic properties of a type-II superconducting wire subject to the concomitant action of an ac transport current and an oscillating transverse magnetic field in synchronous and asynchronous regimes are thoroughly studied under the critical state approach. Relative double frequency effects between the electromagnetic excitations have been explored for the asynchronous cases. Outstandingly, the occurrence of this event can drastically alter the efficiency of the superconducting wire by increasing the ac losses, contrary to the prediction of a reduction in the ac losses when a relative phase shifting is considered. Likewise, striking magnetization loops and remarkable differences to the ac losses predicted by simplified analytical approaches are reported. For cyclic regime, the time-dependent distribution of local current density, the density of power dissipation, and the components of the magnetic flux density are shown in a wide number of cases. Multiply connected domains are revealed for the flux front profiles via the time-dependent consumption of the magnetization currents by effect of the injected transport current lines. Finally, we have shown that the strong localization of the power density and the recently envisaged low pass filtering effect in the wire's magnetic response is only affordable when both electromagnetic excitations evolve synchronous. (C) 2013 AIP Publishing LLC. [http://dx.doi.org/10.1063/1.4804931]
\end{abstract}

\section{INTRODUCTION}

Type-II superconducting wires are deemed promising elements for large-scale technological applications such as power transmission cables, magnet systems for large particle accelerators, and magnetic-based medical techniques such as MRI. The usefulness of this kind of technology is straightforwardly linked to the local electromagnetic response of the superconductor under variations of the ambient magnetic field and the customary condition of transport-current. Special interest is paid on determining the value of the maximum dissipation-free current and characterizing the mechanisms of reduction of electric power dissipation due to alternating fields and/or alternating current flow (commonly called ac losses).

Although the hysteretic losses associated with a superconducting material are noticeably smaller than the range of power dissipated in normal metals, from the practical point of view, the superconducting technology is still not so attractive to replace the power technology based in copper wires, because in order to keep the temperature of the superconductor below $T_{c}$, heat removal requires a sophisticated and costly cryogenic system. Thus, in order to make the superconducting devices more attractive and competitive with respect to other technologies, it is of utter importance to understand, predict, and eventually reduce the ac losses of superconducting wires under practical configurations.

\footnotetext{
a) Author to whom correspondence should be addressed. Electronic mail: hsruizr@gmail.com
}

Major features of the macroscopic electromagnetic behavior of type-II superconducting wires have been captured in Bean's model of the critical state (CS). ${ }^{1}$ In this framework, macroscopic magnetization currents of density $\mathbf{J}$ are induced within the superconductor during variations of the magnetic flux which accordingly redistribute themselves to screen the penetrating flux within the sample. Their magnitude adopts the critical value $J_{c}$ at a given temperature and specified field. Although simple for idealized configurations, the electrodynamics underlying Bean's model becomes cumbersome when realistic configurations are addressed. Thus, penetration of magnetic flux must be typically obtained by more or less sophisticated numerical methods.

In a superconducting slab or a large cylinder (radius much smaller than their length) exposed either to a parallel magnetic field or a longitudinal transport current, the magnetic field has only one component, and the analysis of the field and current distribution is simple. ${ }^{1}$ Nevertheless, if the situation is such that the local magnetic field has two components that are functions of two spatial variables, then solving the electromagnetic problem for arbitrary relations of the external excitations becomes more and more complicated. In fact, for real applications of superconducting wires, the scenario is such that a simultaneous field and transport current condition must be satisfied. Then, in addition to the ambient field one has the magnetic field generated by the transport current itself. This is the situation, for instance, in threephase power transmission lines, as well as in superconductor windings where each wire is subjected to the magnetic field of its neighboring wires. Thus, as this is the configuration 
very often met in practice, this paper is devoted to study round superconducting wires under different conditions of transverse magnetic field and transport current flow, with synchronous and asynchronous regimes for their timedependences. The broad number of examples presented in this paper aims to provide a thorough understanding on the main parameters controlling the hysteretic losses in superconducting wires, which could help in the design of new kinds of applications.

The paper is organized as follows. In Sec. II the theoretical framework of the problem is introduced, with special attention to the numerical procedure of our variational approach for the critical state theory. Then, in Sec. III we give a number of explicit examples related to the electromagnetic behavior of filamentary type II superconducting wires under simultaneous ac transport current and oscillating transverse magnetic field, subjected to the condition that both sources present a synchronous action in time (Fig. 1). Premagnetized wires have been also considered and, for a closer connection with the experimental quantities, we present the wire's magnetic moment $(\mathbf{M})$ and the hysteretic losses $(L)$ as a function of the ac external excitations. This section extends our previous results for synchronous excitations. ${ }^{2}$ In addition, in this paper we show the calculated patterns for the local dynamics of the electromagnetic quantities such as the inner distribution of current density $\mathbf{J}$, the components of the magnetic flux density $\mathbf{B}$, the isolevels of the vector potential A (i.e., the lines of magnetic field, here), and the local distribution of the density of power dissipation $\mathbf{E} \cdot \mathbf{J}$, for a broad number of cases. On the other hand, Sec IV addresses a comprehensive study of the electromagnetic response of the superconducting wire when it is subjected to an asynchronous regime on the time-dependence of the oscillating excitations. In particular, double frequency effects have been considered (Fig. 2), where loops with exotic shapes nonconnected to Bean-like structures are outlined. Furthermore, contrary to the generalized assumption that asynchronous sources may attain reductions in the hysteretic losses, we show that as a consequence of considering double frequency effects, noticeably increase of the hysteretic losses may be found. Finally, Sec. V is devoted to discuss the main results of this work.

\section{GENERAL CONSIDERATIONS AND THEORETICAL STATEMENTS}

This section is devoted to introduce the theoretical background that justifies the critical state concept and the implementation of the variational method as a valid procedure for obtaining the response of superconducting wires subjected to the concomitant action of transverse magnetic field and electric transport current. As stated above, the major features for determining the electromagnetic performance of type-II superconductors have been captured by Bean in the phenomenological model of the critical state. ${ }^{1}$ In this macroscopic approach of coarse-grained fields, the electromagnetic quantities are supposed to be taken over a volume containing a big enough number of vortices. For simplified (1D) configurations, Ampere's law $d B / d x=\mu_{0} J$ is solved with some prescription for the current density $\left(|J| \leq\left|J_{c}\right|\right)$ under continuity boundary conditions that incorporate the influence of the sources. However, when realistic configurations are addressed in a fore, such as a strip with finite thickness or a bulk superconducting cylinder exposed to a transverse magnetic field, exact analytical solutions have not been obtained.

Already in early calculations as those performed in 1970 s and 1980s various analytical simplifications for the modelling of superconducting wires under transverse magnetic field and cross section dimensions much lesser than their length have often been used, by assuming that the flux front can be approached through the use of sinusoidal or elliptical functions. ${ }^{3-5}$ A comprehensive review of the aforementioned methods is found in Refs. 6 and 7. Just in extreme cases such as infinitely thin strips, exact analytical solutions

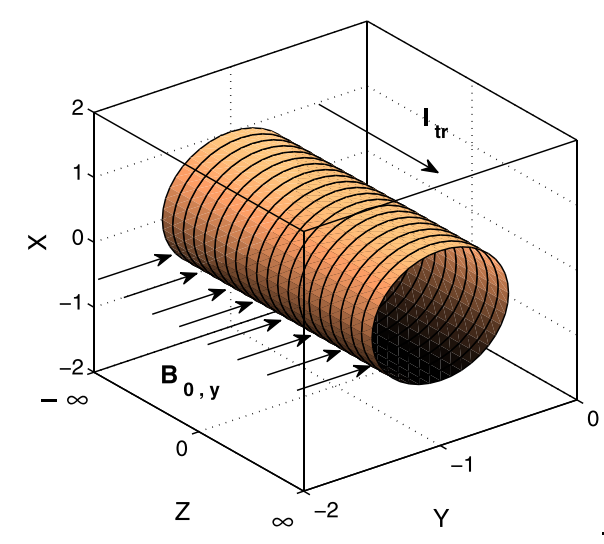

$\mathrm{B}_{\mathrm{a}}:-1.0-2.0-4.0-6.0$

$\mathrm{I}_{\mathrm{a}}:---0.25---0.5---0.75---1.0$
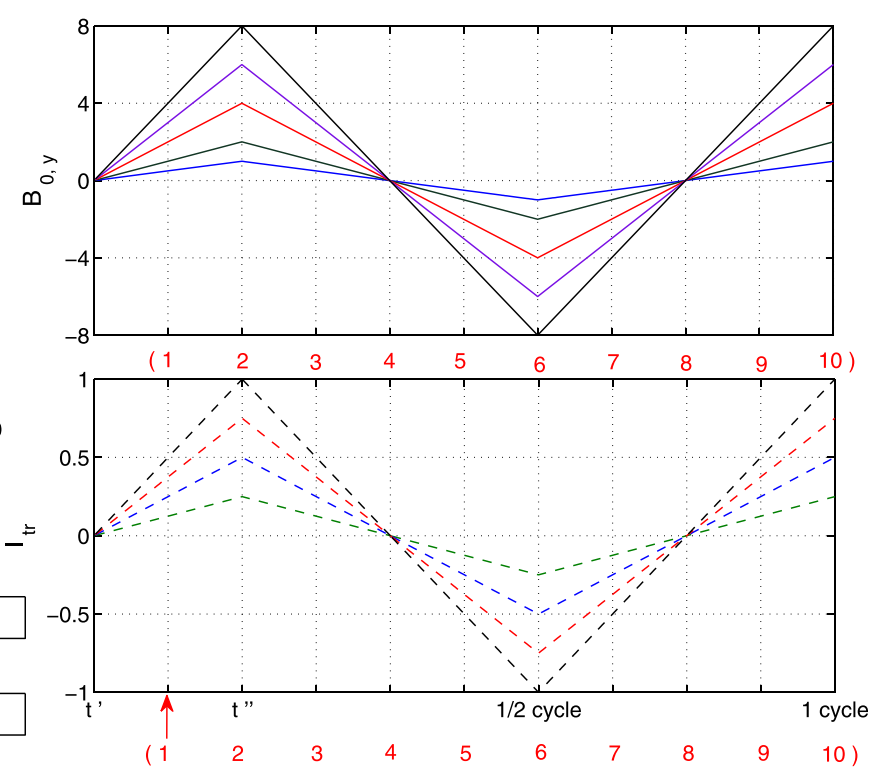

FIG. 1. Sketch of some of the experimental processes analyzed along Sec. III of this paper. Here, a cylindrical SC wire of radius R is subjected to synchronous oscillating excitations $B_{0, y}$ and $I_{t r}$, of amplitudes $B_{\mathrm{a}}$ and $I_{\mathrm{a}}$. Hereinafter, units are $\left(\mu_{0} / 4 \pi\right) R J_{c}$ for $B_{0, y}$, and $I_{c} \equiv \pi R^{2} J_{c}$ for $I_{t r}$. 
(a)
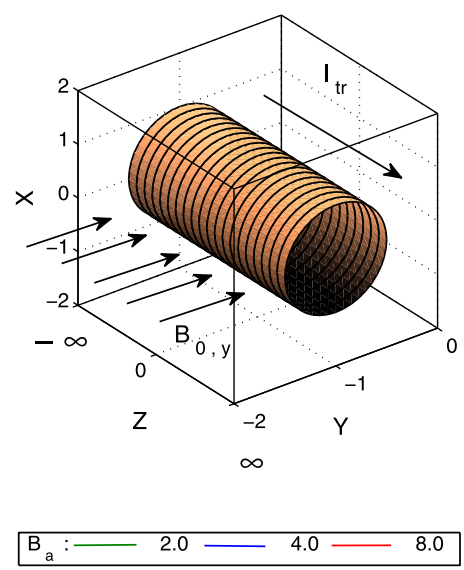

\begin{tabular}{lll}
\hline $\mathrm{I}_{\mathrm{a}}:-2.0-$ & $-0.0-8.0$ \\
\hline
\end{tabular}
( b )
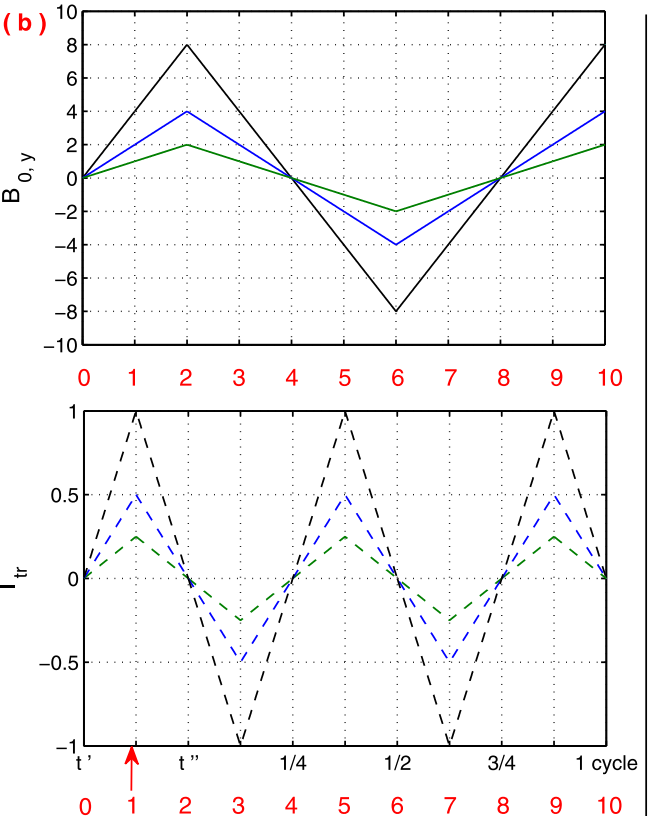
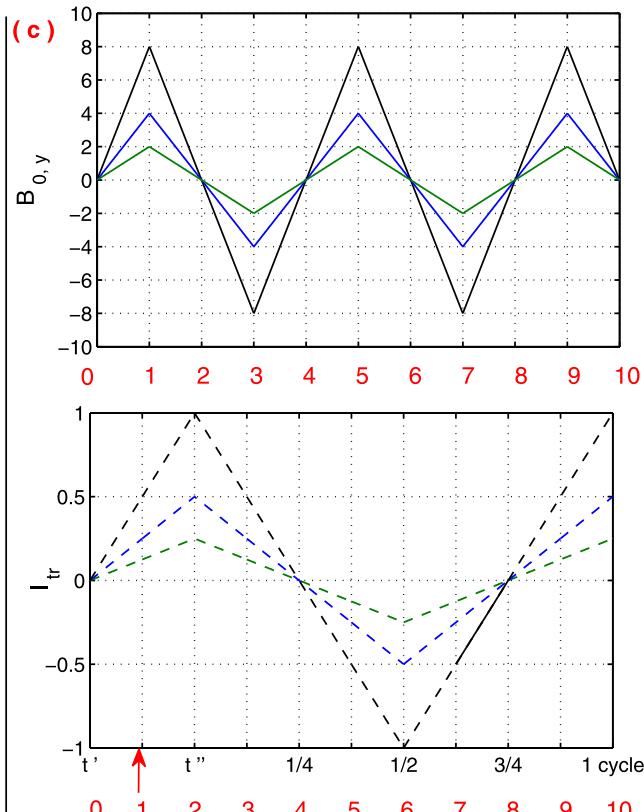

FIG. 2. Sketch of some of the experimental processes analyzed along Sec. IV of this paper. Here, a cylindrical SC wire subjected to asynchronous oscillating excitations in the configuration shown in pane (a) is considered according to the temporal processes depicted in panes (b) and (c).

can be achieved. ${ }^{8-12}$ Also, analytical expressions for the magnetic field and current distributions within the critical state model for hollow superconducting tubes of thickness much smaller than their external radius have been recently reported. ${ }^{13}$ However, when the problem is such that the superconductor is simultaneously subjected to a transport current, asymmetric deformations of the flux free region appear, and analytical approaches are not conceivable. Then, the implementation of optimization techniques and numerical analysis becomes a practical method for handling intricate configurations where simultaneous alternating transport current and transverse applied field occur. Thus, in a pioneering work, a consistent implementation of a variational approach allowed Ashkin to trace out the true structure of the partly flux-penetrated state of a superconducting wire subject to a transverse magnetic field, ${ }^{14}$ whose results were confirmed by various numerical and analytical calculations. $^{15-21}$

At this point, it is worth of mentioning that currently the most popular trend in the analysis of magnetic flux dynamics in superconductors is the numerical simulations implementing finite-element methods in conjunction with nonlinear power-law voltage-current characteristics ${ }^{22-25}$ or new formulations of the $\mathrm{CS}^{26-30}$

\section{A. Variational statement}

Our numerical solution of the CS problem ${ }^{29,30}$ relies on the resolution of the Maxwell equations by means of an equivalent variational statement so as to avoid the numerical integration of this set of differential equations. Thus, we aim at incorporating Ampère's and Faraday's laws by formally integrating the Euler-Lagrange equations

$$
\mu_{0} \mathbf{J}_{l+1}-\nabla \times \mathbf{B}_{l+1}^{*}=0
$$

and

$$
\mu_{0} \nabla \times \mathbf{p}_{l}^{*}+\mathbf{B}_{l+1}^{*}-\mathbf{B}_{l}=0 .
$$

They are considered as derived through arbitrary variations of two variables: the time-discretized local magnetic induction field $\mathbf{B}_{l+1}$ and of a certain Lagrange multiplier $\mathbf{p}_{l}$, i.e., $\delta \mathbf{B}_{l+1} \equiv \mathbf{B}_{l+1}^{*}-\mathbf{B}_{l+1}$ and $\delta \mathbf{p}_{l} \equiv \mathbf{p}_{l}^{*}-\mathbf{p}_{l}$. Starred quantities denote the stationary configuration that solves the problem.

Physically, recall that the induction field $\mathbf{B}$ modifies the ambient magnetic field $\mathbf{H}$ due to microscopic current loops within the material media. However, in the critical state regime the use of the linear relation $\mathbf{B}=\mu_{0} \mathbf{H}$ is allowed; henceforth, we will refer to magnetic field where either or both fields apply. Eventually, the Lagrange multiplier, $\mathbf{p}_{l}$, will be basically identified with the electric field of the problem.

Mathematically, going into more detail, let us consider a small path step $\delta t$, from some initial profile of the magnetic field $\mathbf{B}_{l}(\mathbf{r})$ to a final profile $\mathbf{B}_{l+1}(\mathbf{r})$, and the corresponding $\mathbf{J}_{l}(\mathbf{r})$ and $\mathbf{J}_{l+1}(\mathbf{r})$. Defining $\Delta \mathbf{B}=\mathbf{B}_{l+1}-\mathbf{B}_{l}$, both configurations can be considered to be connected by a steady process performing a small linear step, such that $\mathbf{B}_{l+1}=\mathbf{B}_{l}+s \Delta \mathbf{B}$ with $s \in[0,1] \delta t$. Recalling that the initial condition fulfills Ampere's law $\nabla \times \mathbf{B}_{l}=\mu_{0} \mathbf{J}_{l}$, as well as $\nabla \cdot \mathbf{B}_{l}=0$ and $\nabla \cdot \mathbf{J}_{l}=0$, the time-averaged Lagrange density, whose action gives way to the above equations (1) and (2) is

$$
\mathcal{L}=\frac{1}{2}|\Delta \mathbf{B}|^{2}+\mathbf{E} \cdot\left(\nabla \times \mathbf{B}_{l+1}-\mathbf{J}_{l+1}\right) \delta t .
$$

Here, we have introduced the critical state electric field through $\mathbf{p}=\mathbf{E}_{c s} \delta t$. This quantity must be properly defined by the imposed material law $\mathbf{E}(\mathbf{J})$.

In our case, the critical state concept enters by declaring that there must be a region $\Delta_{\mathbf{r}}$ within the $\mathbf{J}$ space such that the physically admissible current density remains within. Eventually, and enforcing the quasistatic form of Ampère's law we end up with the variational statement 


$$
\operatorname{Minimize} \int_{\Re^{3}} \frac{1}{2}|\Delta \mathbf{B}|^{2}
$$

with the prescription that the minimization procedure accomplishes the boundary conditions imposed by the prescribed sources and the material law $\mathbf{J} \in \Delta_{\mathbf{r}}$.

For consistency, we must mention that, in some cases, charge conservation is not automatic in the above quasistatic formulation that relies on the evaluation of $\nabla \times \mathbf{E}$ and ignores the appearance of local charge $\left(\nabla \cdot \mathbf{E}=-\nabla^{2} \Phi \neq 0\right)$. Thus, in those cases when an intrinsic electromagnetic source must be considered, i.e., $\nabla \Phi \neq 0$, the global set of variables must be further constrained. For example, if the superconductor is carrying a transport current $I_{t r}$ flowing across the section $s$, one has to mandatorily consider the external condition

$$
\int_{s} \mathbf{J} \cdot \hat{\mathbf{n}} d s=I_{t r}
$$

and also update the fields so as to satisfy the physical relation $\mathbf{E} \cdot \mathbf{J}=0$ at those points where the magnetic flux does not vary. As it was said in Ref. 2 this can be accomplished by means of using a calibrated potential $\tilde{\mathbf{A}}$, accordingly to the statement $\mathbf{E}=-\partial_{t} \mathbf{A}-\nabla \Phi \equiv-\partial_{t} \tilde{\mathbf{A}}$.

\section{B. Numerical method for the cases of interest}

Hereafter, we will consider the action of an impressed ac transport current $I_{t r}(t)$ directed along the main axis of an infinite type-II superconducting cylinder of radius $\mathrm{R}$ and cross section $\Omega$ lying in the $x y$-plane, and an oscillating transverse magnetic field of flux density $\mathbf{B}_{0}(t)=\left(0, B_{y}, 0\right)$. In this case, the cumbersome analysis of the intrinsic anisotropy effects ${ }^{29}$ may be straightforwardly avoided as, by symmetry, the current streamlines preserve only one direction (perpendicular to the applied magnetic field), which means a significant reduction of the computational time.

Going into detail, we are enabled to discretize the crosssection area $(\Omega)$ through a collection of points $\left(\mathbf{r}_{i}\right)$ depicting the straight infinite elementary filaments fulfilling the condition $r_{i} \in \Omega$. Thus, for a sufficiently large mesh, a uniform current density can be assumed within each elementary wire such that $I_{i}=J_{i} s_{i}$ with $s_{i}$ the cross-sectional area of the filament. Then, the problem can be straightforwardly written in terms of local contributions of the vector potential $A_{i}\left(r_{i}\right)$ accordingly to cylindrical filaments of section $s_{i}=\pi a^{2}$ with $a \ll R$. Therefore, the vector potential of each filament $\left(A_{i}\right)$ splits up into two expressions, one within the filaments of radius $a$ (i.e., $\forall r_{i}<a$ )

$$
A_{i}\left(r_{i} \in s_{i}\right)=\frac{\mu_{0}}{4 \pi} J_{i}\left[2 \pi a^{2} \ln (a)-\pi\left(a^{2}-r_{i}^{2}\right)\right]+C_{1}
$$

and one outside the filament (i.e., $\forall r_{i j} \neq 0$ )

$$
A_{i}\left(r_{j} \notin s_{i}\right)=-\frac{\mu_{0}}{4 \pi}\left[J_{i} \ln \left(r_{i j}^{2} / a^{2}\right)\right]+C_{2} .
$$

Here, $r_{i j}$ denotes the distance between the centers of filaments $i$ and $j$, and $C_{1}$ and $C_{2}$ are arbitrary integration constants, one of them determined by continuity at $r_{i}=a$ and the other one can be absorbed in a global constant for the whole section $\Omega(C$ in what follows). In fact, as has been established in Sec. II A, $C$ relates to the supply of electrostatic charge. More specifically, in the long wire geometry, one has $C \hat{\mathbf{z}}=-\nabla \Phi$, i.e., $\mathbf{E}=-\partial_{t} \mathbf{A}-\nabla \Phi=-\partial_{t} \mathbf{A}+C \hat{\mathbf{z}}$.

Thus, in the quasi-steady regime (excellent approximation for the large scale application frequencies) the discrete form of Faraday's law $\left[\delta \mathbf{B}_{i}=-\nabla \times \mathbf{E}_{i}\left(\mathbf{J}_{i}\right) \delta t\right]$ in a mesh of circuits that carry the macroscopic electric current density $J_{i}$ is correctly obtained by minimizing the action of the averaged field Lagrangian of density $\mathcal{L}=[\mathbf{B}(t+\delta t)-\mathbf{B}(t)]^{2} / 2$, whilst $\mathbf{E}$ must be refined step by step through the constant $C$. Eventually, standard electromagnetic manipulations and the concept of mutual inductance between circuits allow to convert Eq. (4) into a discretized form. The quantity to be minimized transforms to the functional

$$
\frac{1}{2} \sum_{i, j} I_{i, l+1} \mathrm{M}_{i j} I_{j, l+1}-\sum_{i, j} I_{i, l} \mathrm{M}_{i j} I_{j, l+1}+\sum_{i} I_{i, l+1} \Delta A_{0}
$$

with $\left\{I_{i, l+1}\right\}$ the set of filaments with unknown current for the time step $l+1, A_{0}$ the vector potential related to nonlocal sources, and $M_{i j}$ the mutual inductance matrix between filaments $i$ and $j$. Accordingly to Eqs. (6) and (7), for filaments of cylindrical cross section $s_{i}$ centered at the positions $r_{i} \in \Omega$ and subject to uniform distributions of current density $J_{i} \in s_{i}$, the inductance matrices can be defined as

$$
\mathbf{M}_{i j}= \begin{cases}\frac{\mu_{0}}{8 \pi}, & \forall r_{i}=r_{j} \in \Omega \\ -\frac{\mu_{0}}{4 \pi} \ln \left(r_{i j} / a\right), & \forall r_{i} \neq r_{j} \in \Omega .\end{cases}
$$

For our cases of interest, $A_{0}$ corresponds to the magnitude of the vector potential produced by a uniform transverse magnetic field $\mathbf{B}_{0}$, which can be calculated from the components of the vectorial expression

$$
\mathbf{A}_{0}\left(\mathbf{r}_{i}\right)=\mathbf{B}_{0} \times \mathbf{r}_{i}
$$

Furthermore, when required, optimization must to be performed under the restriction of applied transport current, i.e.,

$$
\sum_{i \in \Omega} I_{i}=I_{\mathrm{tr}}
$$

and the physically admissible solutions have to be constrained by the CS material law for the current density that in this case reads $\left|J_{i}\right| \leq J_{c}$.

Minimization is done under prescribed sources $\left(B_{0}, I_{t r}\right)$ for the time step $l$ and the above material law, and as result of the optimization procedure one gets the distribution of current filaments along the cross section of the superconducting sample at the time step $l+1$. Eventually, the vector potential can be evaluated in the whole space by superposition of Eqs. (6), (7), and (10). Then, one may plot the magnetic flux lines as the isolevels of the total vector potential $\mathbf{A}$, and the components of the magnetic flux density can be evaluated according to its definition $\mathbf{B}=\nabla \times \mathbf{A}$. 
Furthermore, in order to achieve a closer connection with experiments the sample's magnetic moment per unit length is calculated by means the vectorial expression

$$
\mathbf{M}=\frac{1}{2} \int_{\Omega} \mathbf{r} \times \mathbf{J} d \Omega
$$

and the hysteretic ac losses per unit time and volume $(\Phi)$ for cyclic excitations of frequency $\omega$ can be calculated by integration of the local density of power dissipation $(\mathbf{E} \cdot \mathbf{J})$ as follows:

$$
L=\omega \oint_{f . c .} d t \int_{\Phi} \mathbf{E} \cdot \mathbf{J} d \Phi
$$

Here, f.c. denotes a full cycle of the time-varying electromagnetic sources. Recall that, under the critical state framework, Joule heat release may be calculated by $\dot{L}=J_{c} E$ as overcritical flow $\left(J>J_{c}\right)$ is neglected because instantaneous response is assumed.

For concluding this section, some technical details are worth of mention as far as concerns our numerical procedure. On the one hand, the mesh utilized for the whole set of calculations presented along this paper is defined in terms of a rectangular grid with filaments equally distanced under the prescribed condition $r_{i j} \geq 2 a$ to satisfy Eq. (9). The number of filaments which have been considered to fill out the cross section of the superconducting cylinder is 3908 . However, owing to the planar symmetry of the problem one is allowed to reduce the number of variables to 1954 (i.e., 977 filaments per quadrant), which is still a large number because the objective function to be minimized is highly nonlinear. To be more specific, the number of quadratic terms in Eq. (8) involves minimizing the action of 1910035 elements, i.e., the sum of elements produced by the mutual inductance terms between filaments $(977 * 977 * 2)$, and 977 linear elements.

Applications of the above statements are developed along Secs. III and IV in a systematic study of infinite cylindrical wires, under a wide variety of experimental conditions with synchronous and asynchronous regimes for the oscillating electromagnetic excitations.

\section{SYNCHRONOUS EXCITATIONS}

The first conceptualization of the problem of superconducting wires under configurations of simultaneous alternating current and applied magnetic field was provided in 1966 by Hancox, ${ }^{31}$ who studied the ac losses through simplified analytical methods for determining the flux front profile in an infinite slab subjected to a field applied parallel to the direction of the injected transport current. This work went almost unnoticed for over a decade, until a similar approach was proposed in 1979 by Carr. ${ }^{32}$ Then, the same kind of experimental configurations but for monotonic rates of the experimental sources $\left(B_{0}, I_{t r}\right)$ has been studied since the $1990 \mathrm{~s}$, under the assumptions of very thin superconducting strips to allow different analytical considerations. ${ }^{11,12,33}$ However, in more realistic situations, where the cross section of the superconducting sample cannot be reduced to one dimension, the use of exact analytical methods is not feasible. Thus, the use of numerical methods as the described in Sec. II becomes in the more attainable procedure for the forecast and understanding of the electromagnetic observables such as the magnetization curves and the ac power density losses.

It is worth mentioning that despite the fact that there is a significant number of works assuming isolated superconducting wires of diverse geometries, mainly strips subjected to synchronous excitations, ${ }^{22,24,32-52}$ a thorough study of cylindrical superconducting wires is still absent, although some predictions for synchronous excitations were recently reported in Ref. 2. This section aims to extend the aforementioned letter in order to constitute a step forward in the understanding of the electromagnetic observables and the local effects associated with the ac losses, through a comprehensive study of the local electrodynamics of superconducting wires under the combined action of oscillating magnetic field $B_{0, y}$ in phase with the impressed ac transport current $I_{t r}$, both with the same oscillating frequency, i.e., fully synchronous excitations (see Fig. 1). Eventually, premagnetized wires will be also considered. Premagnetized wires subjected to synchronous or asynchronous sources may be found in superconducting multicoils for the production of high magnetic fields,${ }^{53}$ accelerator magnet technologies, ${ }^{53-55}$ and superconducting magnetic energy storage systems. ${ }^{56}$

\section{A. Flux penetration profiles}

In order to achieve a clear understanding of the local dynamics of the profiles of current density flowing across the superconducting wire, let us introduce an intuitive statement whose aim is splitting the sort of filaments with current density $\pm J_{c}$ in terms of two different groups depending on the nature of the source. On the one hand, we will speak about magnetization currents when the local patterns of current density are produced by only the excitation of magnetic flux density $B_{0, y}(t)$. On the other hand, we will refer to the injected current lines when the set of filaments is clearly defined by the global constraint $\sum_{i} I_{i}(t) \equiv I_{t r}(t)$ [Eq. (11)]. When the action of isolated sources is conceived, it is well known that the distribution of screening currents preserves a well defined symmetry according to the shape of the flux front profiles. For instance, quasi-elliptical shapes for the distributions of magnetization currents, or circular for the injected transport current lines. However, for simultaneous application of both sources (Fig. 1), the consumption of the magnetization currents by the injected current lines distorts the axisymmetric orientation of the flux-front, by displacing the current free core to one side (left in this case) during the monotonic branch (Figs. 3 and 4).

Certainly, the flux front profile in the initial stage penetrates from the surface as the intensity of the external excitations $\left[B_{0, y}(t), I_{t r}(t)\right]$ increases. For low magnetic fields compared with the value $B_{p}=\left(2 \mu_{0} / \pi\right) J_{c} R$ which addresses the full penetration condition for $I_{t r}(t)=0$, the profiles of current density are rather similar to those obtained for $B_{0, y}=0$. The basic difference is that, in our case, the center of the current free core moves towards the left (Fig. 3). In fact, 

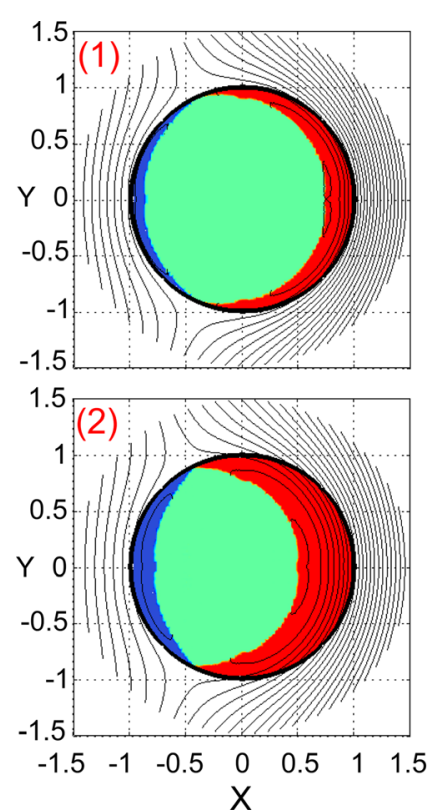
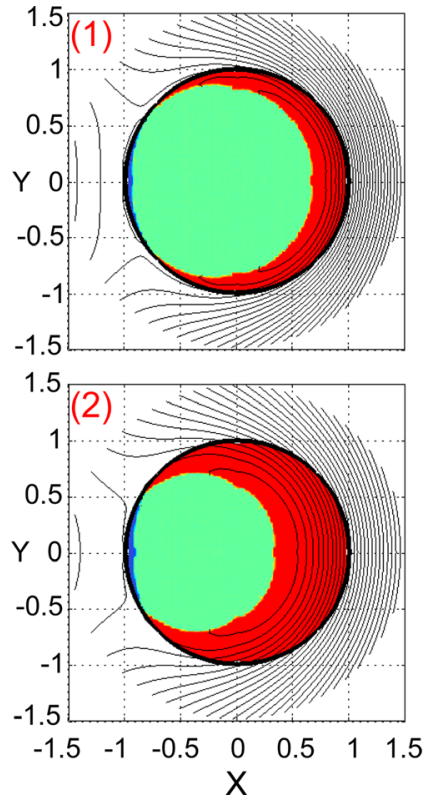
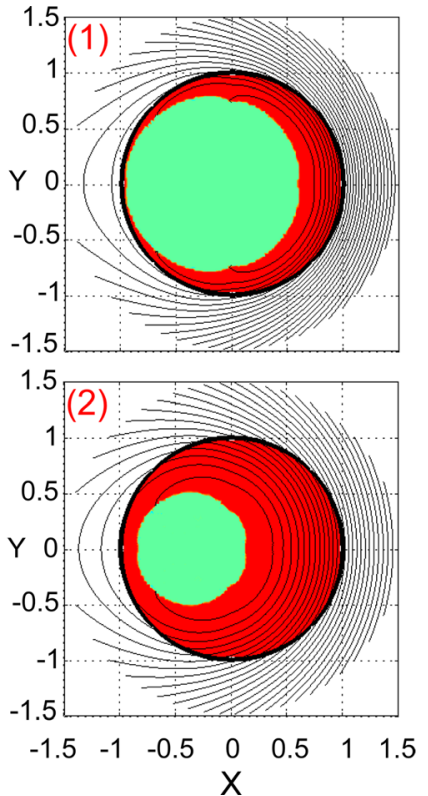
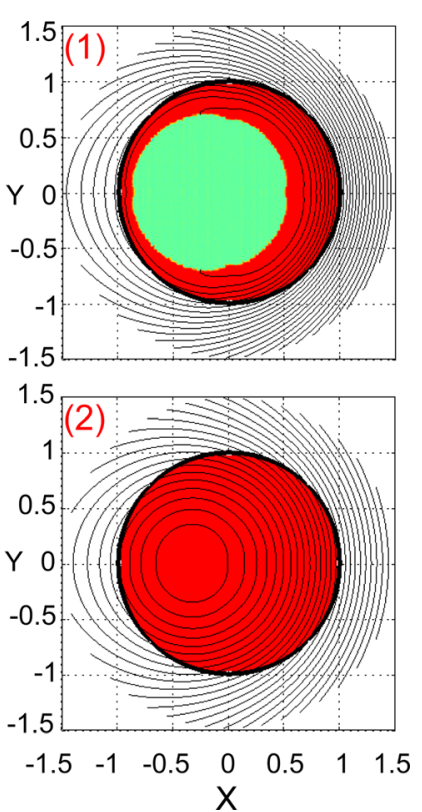

FIG. 3. Evolution of the magnetic flux lines and their corresponding profiles of current with simultaneous oscillating sources $\left(B_{0, y}, I_{t r}\right)$ of amplitudes $B_{\mathrm{a}}=2$ (low field) and, 1 st column: $I_{\mathrm{a}}=0.25,2$ nd column: $I_{\mathrm{a}}=0.5,3$ rd column: $I_{\mathrm{a}}=0.75$, and 4 th column: $I_{\mathrm{a}}=1$. Subplots are labeled according to the monotonic branch of the experimental processes depicted in Fig. 1. For visualizing the electromagnetic response in the following branches (cyclic response), including the intensity of the components of magnetic flux density, reader is advised to see the supplementary material (Figs. 1-3 (Ref. 57)).

if the intensity of the transport current is high enough, the flux front becomes nearly circular (current-like). Then, the distribution of screening currents may be understood as the straightforward overlapping of the profiles of current density for isolated sources, i.e., those referred as magnetization currents $\left[B_{0, y}(t), I_{t r}=0\right]$ and the injected current lines $\left[B_{0, y}=0, I_{t r}(t)\right]$, plus a displacement of the center of the core devoid of electric current and magnetic flux (green zone) given by the respective difference between the known flux fronts. It should be mentioned that such assumption has been made in Refs. 11 and 12 for calculating the current profiles for thin strips with synchronous excitations $\left[B_{0}(t), I_{t r}(t)\right]$. Nevertheless, it has been proved that even in this simple configuration, the overlapping principle for the flux front tracking may be only fulfilled for high current and low applied field. ${ }^{2,46}$

Likewise, if the applied magnetic field is intense enough as compared to the transport current (see, e.g., left side in Fig. 4) the distribution of screening currents is field-like. Nevertheless, the inherent existence of injected current lines makes it impossible to discern which filaments correspond to the so called magnetization currents, and which are the injected current lines. Certainly, for the monotonic branch of the cyclic excitation and before attaining a full penetration state by the screening currents, the "active" zone (blue) where $I_{i}$ takes the value $-I_{c}$ straightforwardly corresponds to the so called magnetization currents. However, the remaining "active" zone (red) defined by screening currents $I_{i}$ taking the value $I_{c}$ is not spatially symmetric as regards the direction of the applied magnetic field, which means that a certain amount of the magnetization currents are contributing in the same direction as the transport current, whilst another part has been consumed by the injected current lines. As it will be shown below, a parallel effect is that the density of magnetic flux increases in the "active" zone, where the patterns of injected current lines dominate.

For the cyclic processes displayed in Fig. 1 tracking the flux front for synchronous excitation with low magnetic field is intuitive, although following up the components of the magnetic flux density $B_{x}$ and $B_{y}$ is not (Figs. 1-3 in supplementary material ${ }^{57}$ ). For high magnetic fields, ascertaining the distribution of screening currents in the cyclic stage is much more elaborated, as long as the electromagnetic history is not erased by the maximal condition for the amplitude of the ac transport current $I_{\mathrm{a}}=I_{c}$. Actually, if $I_{\mathrm{a}}<I_{c}$ the flux fronts do not overlap to a unique contour line defined by the filaments with current alternating between $I_{c}$ and $-I_{c}$ (Figs. 4-6 in supplementary material $\left.{ }^{57}\right)$. Likewise, describing the evolution of the magnetic flux density is also complicated if one compares them with the simplest cases in which isolated sources are assumed, and even this situation becomes much more complicated if premagnetized wires are considered (Figs. 7-9 in supplementary material ${ }^{57}$ ).

However, although the analysis of the magnetic flux density $B$ is complicated, one of the most outstanding observations when considering synchronous excitations as shown in Fig. 1 is that the local distribution of magnetic field preserves the same kind of pattern along the cyclic stage, independently of the intensity of the external sources. Thus, one can notice that the maximal density of magnetic flux occurs always to the right side of the superconducting wire, which corresponds to the "active" zone where the injected current lines are dominating the system. Concomitantly, substantial distortions of the magnetic flux density outside the wire appear. These are particularly marked when $B_{0, y}$ and $I_{t r}$ go to zero during the excitation.

Remarkably, the strong localization of the inner density of magnetic flux density produces a significant change in the 

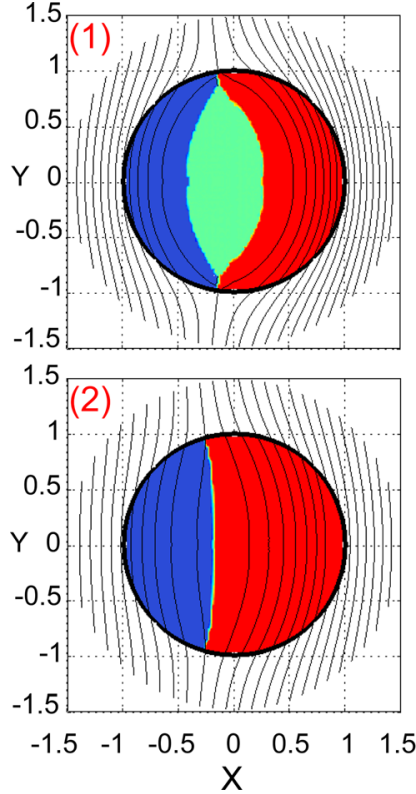
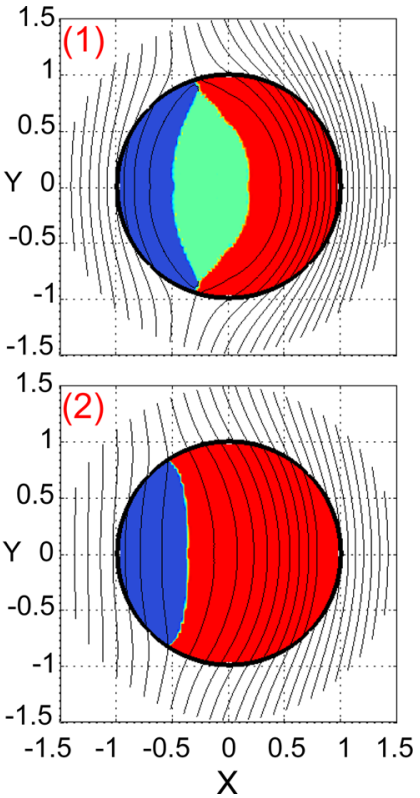
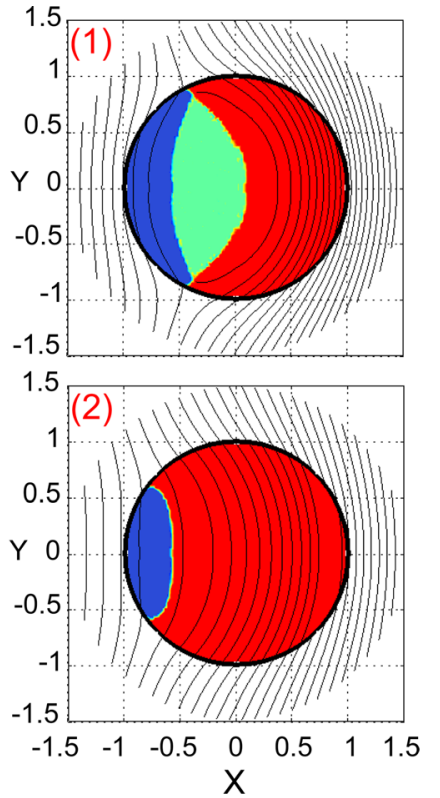
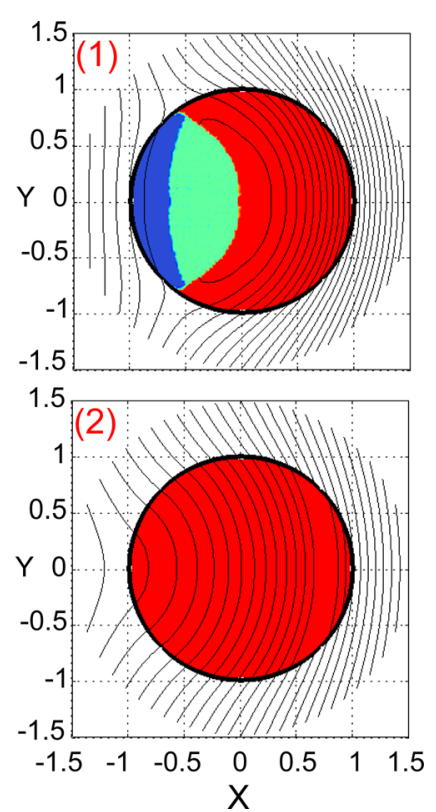

FIG. 4. Evolution of the magnetic flux lines (projected isolevels of the vector potential) and their corresponding profiles of current with simultaneous oscillating sources $\left(B_{0, y}, I_{t r}\right)$ of amplitudes $B_{\mathrm{a}}=8$ (high field) and, 1 st column: $I_{\mathrm{a}}=0.25,2$ nd column: $I_{\mathrm{a}}=0.5$, 3rd column: $I_{\mathrm{a}}=0.75$, and 4 th column: $I_{\mathrm{a}}=1$. Subplots are labeled according to the monotonic branch of the experimental processes depicted in Fig. 1, i.e., label (1) identifies the time-step corresponding to half of the first branch, and (2) the first excitation peak. For visualizing the electromagnetic response in the following branches (cyclic response), including the intensity of the components of magnetic flux density, reader is advised to see the supplementary material (Figs. 4-6 (Ref. 57)).

local distribution of density of power dissipation $\mathbf{E} \cdot \mathbf{J}$ (Fig. 5), which rises from low-value parts (blue) to highvalue parts (red), in such manner that the heat release from the superconducting wire should highly localized too. This asymmetric distribution of power losses remains along the entire cyclic process as long as both excitations evolve synchronous. ${ }^{58}$ Such a pronounced bias unfolding across the wire could increase the probability of quench. For an extended illustration of this phenomenon, we encourage to the readers to see the supplementary material (Figs. 10-12 (Ref. 57)).

\section{B. Magnetic response}

The above described behavior for the local flux distributions gives way to the following features on the magnetic moment response.
For the set of cases displayed in Fig. 1, we have analyzed the dynamics of the magnetic moment component $M_{y}$ as a function of the amplitudes of the electromagnetic sources $B_{\text {a }}$ and $I_{\mathrm{a}}$ (Fig. 6). Results are shown accordingly to the temporal dependence with the synchronous ac excitations (right pane), and also by their dependence with each one of the electromagnetic sources, say $I_{t r}$ (left pane) and $B_{0, y}$ (middle pane). We realize that only for small values of the amplitude of the ac transport current, almost Bean-like loops of $M_{y}$ are obtained. However, as $I_{\mathrm{a}}$ grows we notice a progressive disappearance of the flat saturation behavior for values of $B_{\text {a }}$ higher than $B_{p}$. Actually, the notorious change of sign for the slope of the magnetic moment curve along a monotonic branch of the synchronous ac excitation allows an unambiguous glimpse of the consumption of magnetization lines by effect of injected current lines. Remarkably,
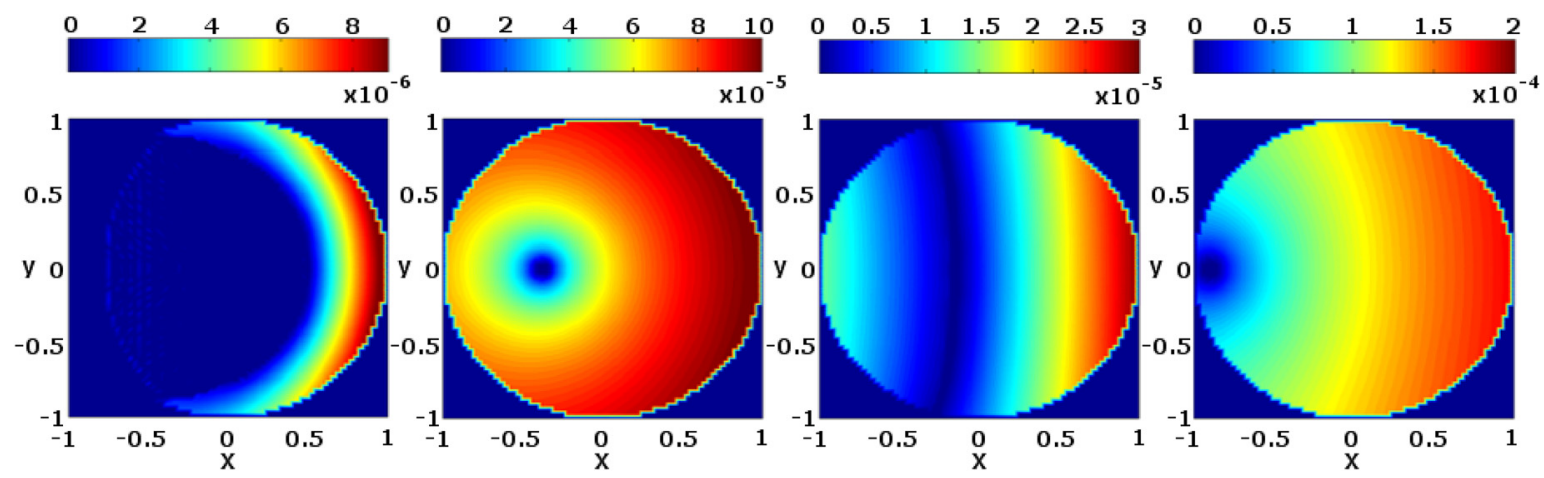

FIG. 5. Local density of power dissipation $\mathbf{E} \cdot \mathbf{J}$ at the time frame of full cycle (step number 10 in Fig. 1$)$ for synchronous oscillating sources of amplitudes: ( 1 st pane) $B_{\mathrm{a}}=8$ and $I_{\mathrm{a}}=0.25$, (2nd pane) $B_{\mathrm{a}}=8$ and $I_{\mathrm{a}}=1.0$, (3rd pane) $B_{\mathrm{a}}=2$ and $I_{\mathrm{a}}=0.25$, (4th pane) $B_{\mathrm{a}}=2$ and $I_{\mathrm{a}}=1.0$. The local dynamics for the aforementioned quantities in the full cyclic process including the initial monotonic branch can be inferred from the supplementary figures for the low-field regime (Fig. 10), high-field regime (Fig. 11), and premagnetized wires (Fig. 12). ${ }^{57}$ Units are $\left(\mu_{0} / 4 \pi\right) R J_{c}$ for $B_{a}, I_{c} \equiv \pi R^{2} J_{c}$ for $I_{a}$, and $\left(\mu_{0} / 4 \pi\right) R^{2} J_{c}^{2} / \delta t$ for $\mathbf{E} \cdot \mathbf{J}$. 

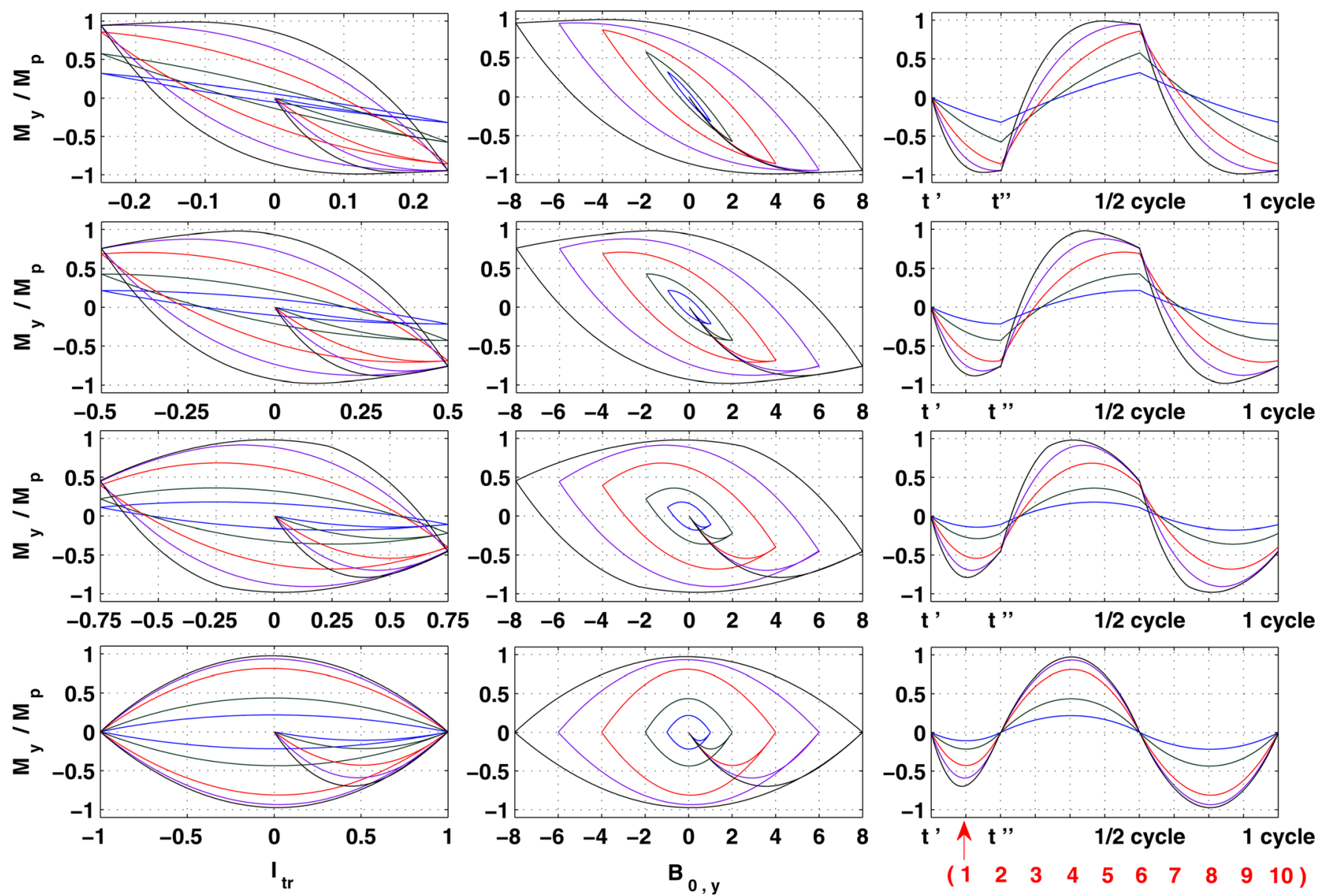

FIG. 6. The dimensionless magnetic moment $M_{y} / M_{p}$ for the synchronous ac excitations displayed in Fig. 1. Curves are shown as function of the injected transport current $I_{t r}$ (left pane), the applied magnetic field $B_{0, y}$ (central pane), or either by their temporal evolution (right pane). In this figure, the amplitudes for the electromagnetic ac sources can be extracted either from color comparison with curves in Fig. 1, or from the respective limits along the abscissas in left and central panes. Units are $\left(\mu_{0} / 4 \pi\right) R J_{c}$ for $B_{0, y}$ and $I_{c} \equiv \pi R^{2} J_{c}$ for $I_{t r}$. Normalization to the full penetration value $M_{p}=2 / 3 R^{3} J_{c}$ is used for $M_{y}$.

this phenomenon ends up with a symmetrization of loops, both as functions of $B_{y}$ and $I_{t r}$, into characteristic lenticular shapes. As a consequence of this process, a distinct low-pass filtering effect comes to the fore which, in the case of the triangular input excitations considered here, yields a nearly perfect sinusoidal (first-harmonic) output signal $M_{y}(t)$.

Interestingly, from the cycles of $M_{y}$, it furthermore appears that a proper determination of the "active" zones depends on the history of the virgin branch, thus bearing witness to the system's memory. For example, a positive slope in the synchronous excitation $B_{y}$ and $I_{t r}$ produces a higher power dissipation in the positive $x$-direction perpendicular to the wire. On the other side, if the superconducting wire has been premagnetized before switching on the synchronous ac excitation $\left[t=t^{\prime}\right]$ at Fig. 7 , the magnetic moment curve is displaced in such a manner that the center of the magnetization loop lies over the master curve for the isolated excitation $B_{0, y}$ at $M_{y}\left(t^{\prime}\right)$, and the nodes move towards the boundaries $B_{0, y}\left(t^{\prime}\right) \pm B_{\mathrm{a}}$.

\section{AC losses}

Regarding the power density losses attained along the premagnetization process, it does not seem to play any role in the calculation of the ac losses (Fig. 8). However, the definition of the flux front profile becomes much more tangled, because multiple domains enclosed by contour lines defined by the screening currents alternating from $I_{c}$ to $-I_{c}$ arise (Fig. 7 in supplementary material ${ }^{57}$ ).

Notwithstanding, the Bean-like magnetic moment curves as the described above, and the ostensible explanation for the distribution of screening currents in terms of the consumption of magnetization lines by the injected current lines, is actually insufficient for the proper interpretation of the actual ac losses produced by synchronous excitations. As we will show below, the feasibility of approaching the total ac loss by overlapping the isolated contributions strongly depends on the relative magnitudes of the ac field $B_{0}$ and the ac transport current $I_{t r}$.

First, let us recall that, the ac losses for a current carrying cylindrical superconducting wire can be easily calculated having in mind that the flux front is defined through axisymmetric circumferences of radius $\tilde{r}=R \sqrt{1-I_{t r} / I_{c}}$, such that

$$
L\left(i_{\mathrm{a}}\right) \equiv \frac{\mu_{0} I_{c}^{2}}{\pi^{2} R^{2}}\left[\left(i_{\mathrm{a}}-\frac{i_{\mathrm{a}}^{2}}{2}\right)+\left(1-i_{\mathrm{a}}\right) \ln \left(1-i_{\mathrm{a}}\right)\right],
$$

where the dimensionless parameter $i_{\mathrm{a}}=I_{\mathrm{a}} / I_{c}$ has been introduced. 

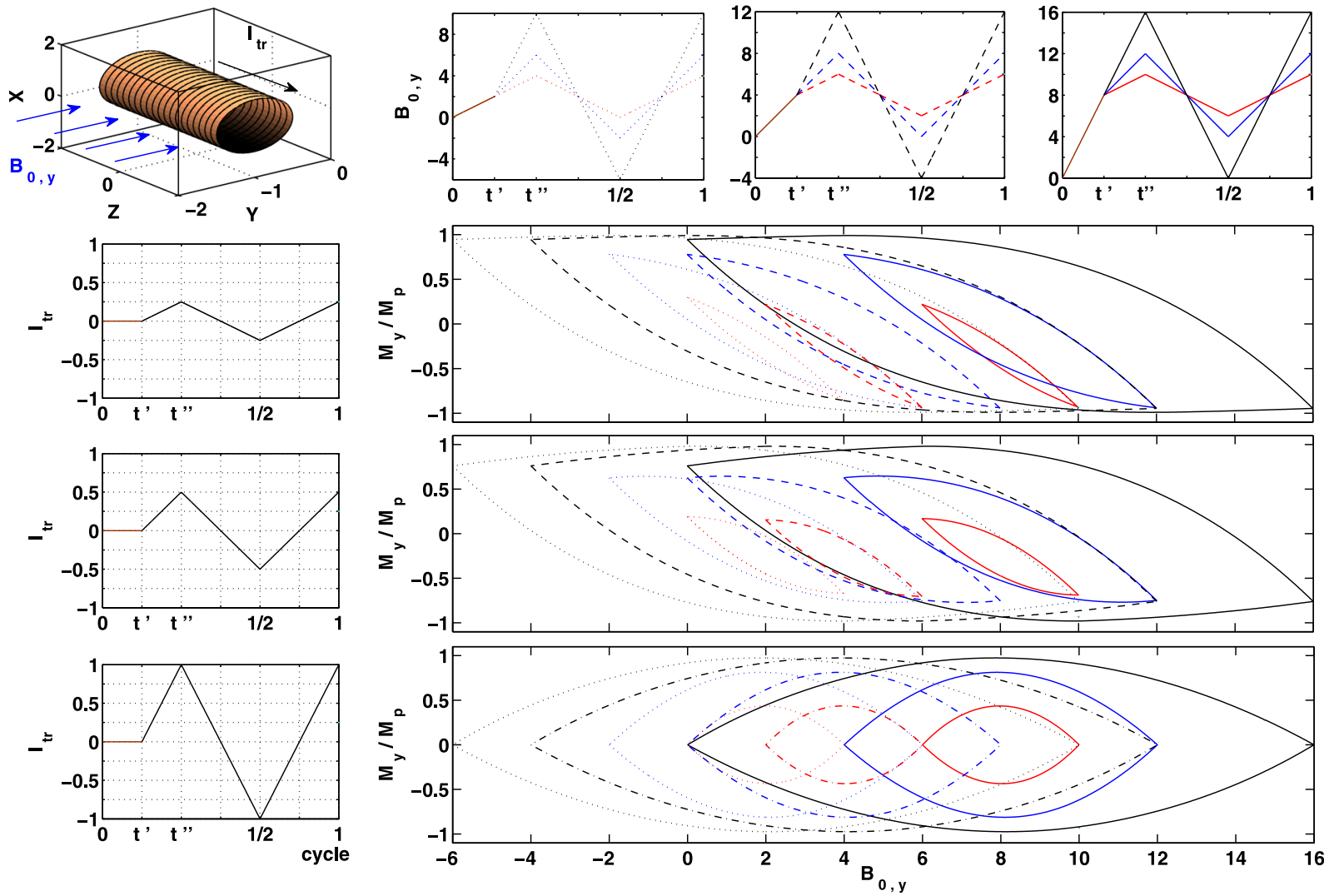

FIG. 7. Dimensionless magnetic moment $M_{y} / M_{p}$ as a function of the applied magnetic field $B_{0, y}$ for cycles of simultaneous ac excitations $B_{0, y}$ and $I_{t r}$ of amplitudes $\left(B_{\mathrm{a}}, I_{\mathrm{a}}\right)$. Although the excitation peak to peak of both sources is assumed to be synchronous, several premagnetized samples have been considered according to: $B\left(t^{\prime}\right)=2, B\left(t^{\prime}\right)=4$ and $B\left(t^{\prime}\right)=8$ (see 1st row), for the time instant when the ac current $I_{t r}\left(t^{\prime}\right)$ is switched on. Regarding to the cyclic process (i.e., from $t^{\prime \prime}$ to 1 ), several cases are shown accordingly to the amplitudes $I_{t r}\left(t^{\prime \prime}\right)=0.25$ (see 2 nd row), $I_{t r}\left(t^{\prime \prime}\right)=0.5$ (see 3 rd row), and $I_{t r}\left(t^{\prime \prime}\right)=1$ (see 4 th row), as well as to $B_{\mathrm{a}}=2$ (dotted lines), $B_{\mathrm{a}}=4$ (dashed lines) and $B_{\mathrm{a}}=8$ (straight lines), respectively.

On the other hand, the monotonic losses produced by magnetization effects may be estimated from the approach of Gurevich et al., ${ }^{7}$ in such manner that the ac losses can be calculated from

$$
L\left(B_{\mathrm{a}}\right) \equiv \frac{8 B_{p}^{2}}{3 \mu_{0}} \begin{cases}b_{\mathrm{a}}^{3}\left(1-\frac{1}{2} b_{\mathrm{a}}\right), & 0<b_{\mathrm{a}} \leq 1 \\ b_{\mathrm{a}}-\frac{1}{2}, & b_{\mathrm{a}} \geq 1\end{cases}
$$

with the dimensionless parameter $b_{\mathrm{a}}=B_{\mathrm{a}} / B_{p}$. Thus, the full penetration state is given by $i_{\mathrm{a}}=1$ or $b_{\mathrm{a}} \geq 1$.
Notoriously, the simplest approach for determining the ac losses of cylindrical superconducting wires subjected to synchronous ac excitations relies in the linear superposition of the separate contributions, $L\left(B_{\mathrm{a}}\right)+L\left(i_{\mathrm{a}}\right)$. Another possibility is to assume that the actual ac losses for synchronous excitations do not strongly differ of the hysteretic losses for superconducting samples carrying a constant transport current $i_{d c}=I_{t r}^{d c} / I_{c}$, and a simultaneous oscillating magnetic field of amplitude $B_{\mathrm{a}}{ }^{7}$ This idea was applied in the analytical approach by Zenkevitch et al ${ }^{59}$ In such a framework, the hysteretic loss for a period is approximated by

$$
L\left(B_{\mathrm{a}}, i_{d c}\right) \equiv \frac{8 B_{p}^{2}}{3 \mu_{0}} \begin{cases}b_{\mathrm{a}}^{3}\left(1-\frac{1}{2} b_{\mathrm{a}}\right), & b_{\mathrm{a}}<i_{d c}^{\dagger} \\ i_{d c}^{\dagger}\left(1-\frac{1}{2} i_{d c}^{\dagger}\right)+\left(1+i_{d c}^{2}\right)\left(b_{\mathrm{a}}-i_{d c}^{\dagger}\right), & b_{\mathrm{a}} \geq i_{d c}^{\dagger},\end{cases}
$$

where we have introduced the dimensionless parameter $i_{d c}^{\dagger}$ $\equiv 1-i_{d c}^{2 / 3}$ and the condition $b_{\mathrm{a}} \geq i_{d c}^{\dagger}$. Thus, $b_{\mathrm{a}} \geq 1$ or $i_{d c}^{\dagger} \equiv 0$, both define a full penetrated sample.

Fig. 8 shows our numerical results for the variation of the actual ac losses of cylindrical SC wires in terms of the amplitude of the synchronous oscillating sources, $L\left(B_{\mathrm{a}}, i_{\mathrm{a}}\right)$ (solid-diamond lines), compared to the customary approaches $L\left(B_{\mathrm{a}}\right)+L\left(i_{\mathrm{a}}\right)$ (dashed lines at the upper pane) and $L\left(B_{\mathrm{a}}, i_{d c}\right)$ (dashed-dotted lines at the lower pane), for four different amplitudes of the ac/dc transport current, and a 


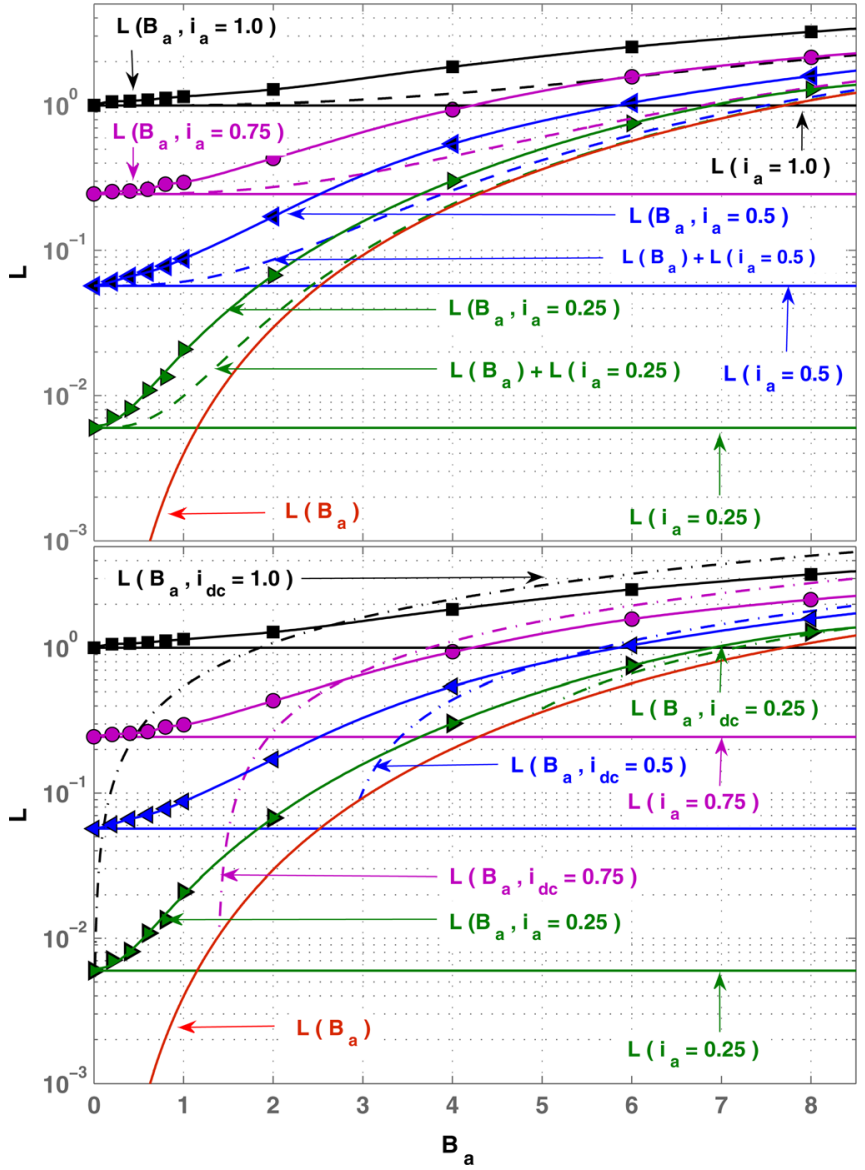

FIG. 8. Hysteretic ac losses per cycle for synchronous ac magnetic flux density and oscillating transport current of amplitudes $\left(B_{\mathrm{a}}, i_{\mathrm{a}}=I_{\mathrm{a}} / I_{c}\right)$ accordingly to Figs. 1 and 7. Numerical results obtained from our variational approach are shown as color solid lines with markers. Comparisons with results from conventional semianalytical approaches are shown for, $(i)$ Top pane: separate excitations $L\left(B_{\mathrm{a}}\right)$ (red solid line) and $L\left(i_{\mathrm{a}}\right)$ (straight color lines), as well as their linear superposition $L\left(B_{\mathrm{a}}\right)+L\left(i_{\mathrm{a}}\right)$ (color dashed lines); (ii) Bottom pane: an ac magnetic field together with a dc transport current of intensity $i_{d c}=I_{\mathrm{tr}}^{\mathrm{dc}} / I_{c}, L\left(B_{\mathrm{a}}, i_{d c}\right)$. Units for losses are $\left(\mu_{0} / 4 \pi\right) \omega R^{2} J_{c}^{2}$.

set of amplitudes of the ac density of magnetic flux. The whole set of results is also plotted in linear scale at Fig. 9.

Comparison reveals the important fact that a linear superposition of contributions due to either type of excitation may be only appropriate for high amplitudes of the magnetic field $\left(B_{\mathrm{a}} \geq B_{p}\right)$ and low currents $\left(i_{\mathrm{a}}<0.25\right)$, or the converse limit, very low magnetic fields $\left(B_{\mathrm{a}} \leq 1\right)$ and extremely high currents $i_{\mathrm{a}} \approx 1$ (i.e., $I_{\mathrm{a}} \lesssim I_{c}$ ); a finding which adds to previous work dealing with a rectangular geometry ${ }^{46}$ and sheds new light on the validity of approximate formulae at the same time. Actually, notice that the actual ac $\operatorname{loss} L\left(B_{\mathrm{a}}, i_{\mathrm{a}}\right)$ is always higher than the instinctive approach $L\left(B_{\mathrm{a}}\right)+L\left(i_{\mathrm{a}}\right)$, whilst the deviation respect to $L\left(B_{\mathrm{a}}, i_{d c}\right)$ strongly depends on the intensity of the electromagnetic excitations. Consequently, approximations such as $L\left(B_{\mathrm{a}}\right)+L\left(i_{\mathrm{a}}\right)$ and $L\left(B_{\mathrm{a}}, i_{d c}\right)$ can drastically under- or overestimate the true losses.

For further understanding of the above behavior, Fig. 9 shows the percentage relation between the actual ac loss, $L\left(B_{\mathrm{a}}, i_{\mathrm{a}}\right)$, and the intuitive approaches, $L\left(B_{\mathrm{a}}\right)+L\left(i_{\mathrm{a}}\right)$ (top pane) and $L\left(B_{\mathrm{a}}, i_{d c}\right)$ (bottom pane), stacked according to the values of $i_{\mathrm{a}}$. On the one side, we note that for the approach
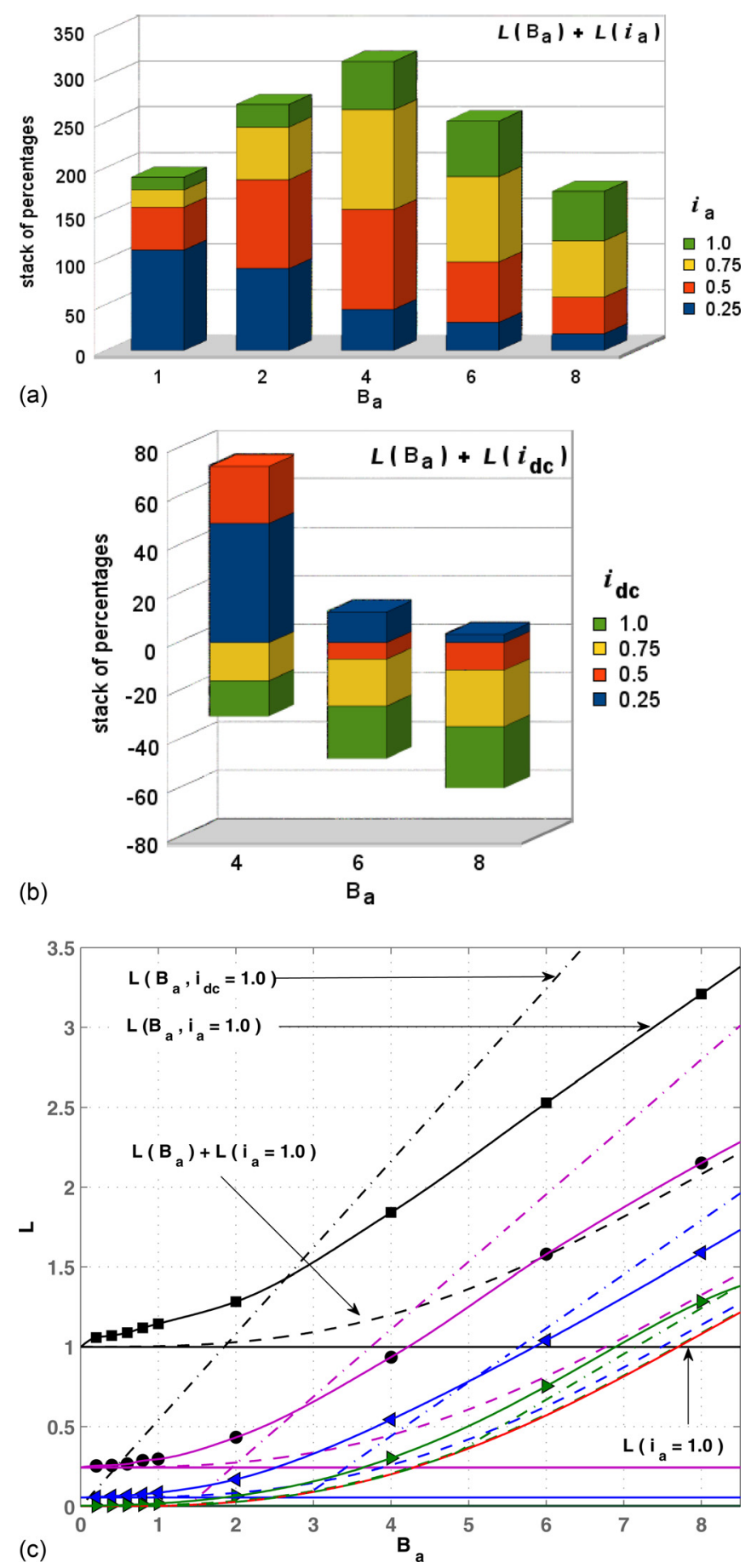

FIG. 9. In the lower pane, the whole set of results shown in Fig. 8 is also plotted in linear scale. The percent change between the actual ac loss $L\left(B_{\mathrm{a}}, i_{\mathrm{a}}\right)$ numerically calculated and the intuitive approaches $L\left(B_{\mathrm{a}}\right)+L\left(i_{\mathrm{a}}\right)$ and $L\left(B_{\mathrm{a}}\right)+L\left(i_{d c}\right)$ is shown in the central and upper panes, respectively.

$L\left(B_{\mathrm{a}}\right)+L\left(i_{\mathrm{a}}\right)$, works well for small values of $i_{\mathrm{a}}$ (e.g., $\left.i_{\mathrm{a}}=0.25\right)$, with the deviation gradually reduced as one increases the amplitude of the magnetic field $B_{\mathrm{a}}$. However, as $i_{\mathrm{a}}$ increases deviations may either reduce (for low values of $B_{\mathrm{a}}$, e.g., $B_{\mathrm{a}}=1$ ) or increase (for high values of $B_{\mathrm{a}}$, e.g., $B_{\mathrm{a}}=8$ ). Moreover, for moderate fields (e.g., $2 \leq B_{\mathrm{a}} \leq 6$ ) percentage deviations first grow as a function of $i_{\mathrm{a}}$ until $i_{\mathrm{a}}=0.5$, and then decrease as $i_{\mathrm{a}}$ approaches the threshold current $i_{\mathrm{a}}=1$. On the other side, the approach $L\left(B_{\mathrm{a}}, i_{d c}\right)$ is not even comparable with the actual ac losses $L\left(B_{\mathrm{a}}, i_{\mathrm{a}}\right)$ for 

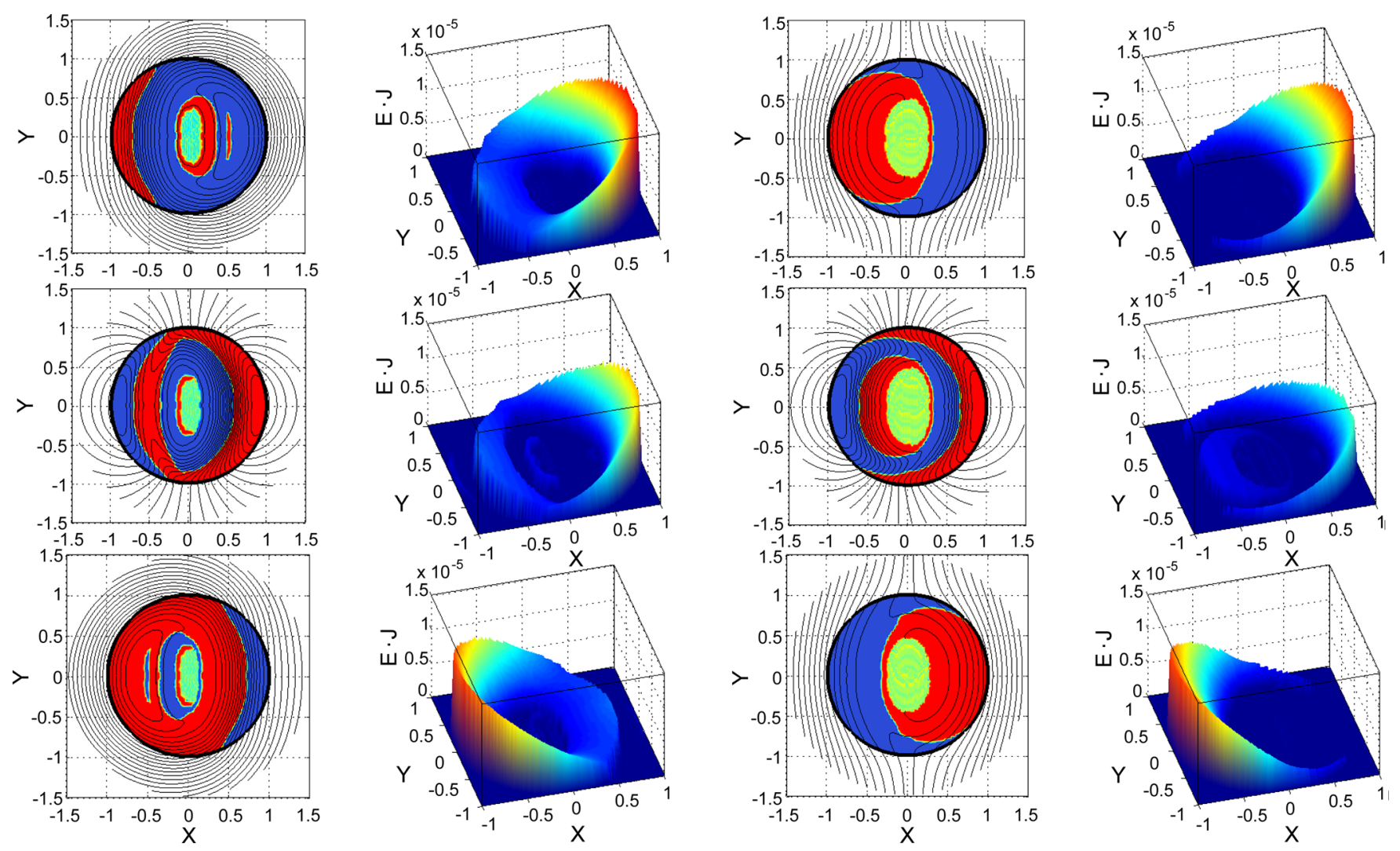

FIG. 10. Evolution of the magnetic flux lines and their corresponding profiles of current with asynchronous oscillating sources $B_{0, y}$ and $I_{t r}$ of amplitudes $B_{\mathrm{a}}=4$ and $I_{\mathrm{a}}=0.5$ (left side into each pane), accordingly to the temporal processes displayed into Fig. 2(b) "left pane herein" and Fig. 2(c) "right pane herein." Also the corresponding profiles for the local density of power dissipation $\mathbf{E} \cdot \mathbf{J}$ are shown (right side into each pane). In particular, in this figure we show the set of results for the last branch of the dominant excitation according to the time-steps marked with the labels (6), (8), and (10) in Fig. 2. More details about the follow up of the electromagnetic quantities along the cyclic process are shown in supplementary material, ${ }^{57}$ Figs. $13-20$. Units are $\left(\mu_{0} / 4 \pi\right) R J_{c}$ for $B_{a}, I_{c} \equiv \pi R^{2} J_{c}$ for $I_{a}$, and $\left(\mu_{0} / 4 \pi\right) R^{2} J_{c}^{2} / \delta t$ for $\mathbf{E} \cdot \mathbf{J}$.

the regime of low magnetic fields (particularly for $b_{\mathrm{a}}<i_{\mathrm{a}}^{\dagger}$ ), a range in which the approximation conceals the effect of $i_{a}$. However, for moderate and high magnetic fields, the percentage ratio between the actual ac loss and the ac loss predicted by the latter approach decrease as $i_{\mathrm{a}}$ increases even reaching negative values. Hence, $L\left(B_{\mathrm{a}}, i_{d c}\right)$ may either overestimate or underestimate the actual ac loss for wires subjected to synchronous oscillating sources. Remarkably, for high amplitudes of the oscillating magnetic flux density $B_{\mathrm{a}}$, we note that a synchronous oscillating transport current of relative amplitude $i_{\text {a }}$ produces a lower amount of hysteretic losses per period than those predicted when the superconducting sample is carrying a constant transport current $i_{d c}$.

\section{ASYNCHRONOUS EXCITATIONS}

In many of the large-scale power applications for superconducting wires, such as windings of motors, transformers, generators and power three-phase transmission lines, the $\mathrm{SC}$ wire is subjected to diverse configurations of electromagnetic excitations, where the ac transport current flowing through and the magnetic field to which the wire is exposed could not fulfill the synchronous conditions referred above (same phase and frequency). Moreover, remarkable experimental differences between the ac loss measured for superconducting wires or tapes with synchronous and asynchronous oscillating transport current and perpendicular magnetic field have been already reported by several authors. ${ }^{43-45}$ In what follows we will address the effects related to the consideration of asynchronous excitations, in which, both sources may be out of phase and apply at different frequencies. However, given the enormous number of possibilities to analyze, we will focus on the influence of double frequency effects over the actual estimation of the ac losses in a superconducting wire, when any of the electromagnetic sources is plugged to some power grid with a double frequency than the other.

\section{A. General considerations}

Our analysis of the ac loss for cylindrical superconducting wires subjected to simultaneous oscillating transport current $\left(I_{t r}\right)$ and perpendicular magnetic flux density $\left(B_{0, y}\right)$ reveals that the total ac loss may be controlled by reducing the inductive magnetic flux density produced by the superposition between the external magnetic field and the contribution by the whole set of screening currents. This can be achieved just by a time shift on one of the ac electromagnetic sources (either $B_{0, y}$ or $I_{t r}$ ) respect to the other, so that the occurrence of the peaks of excitation for each one of the electromagnetic sources is no longer synchronous with the other. Thus, some eccentric branches with opposite temporal derivatives appear between two consecutive peaks of the dominant excitation (i.e., the excitation with lower 

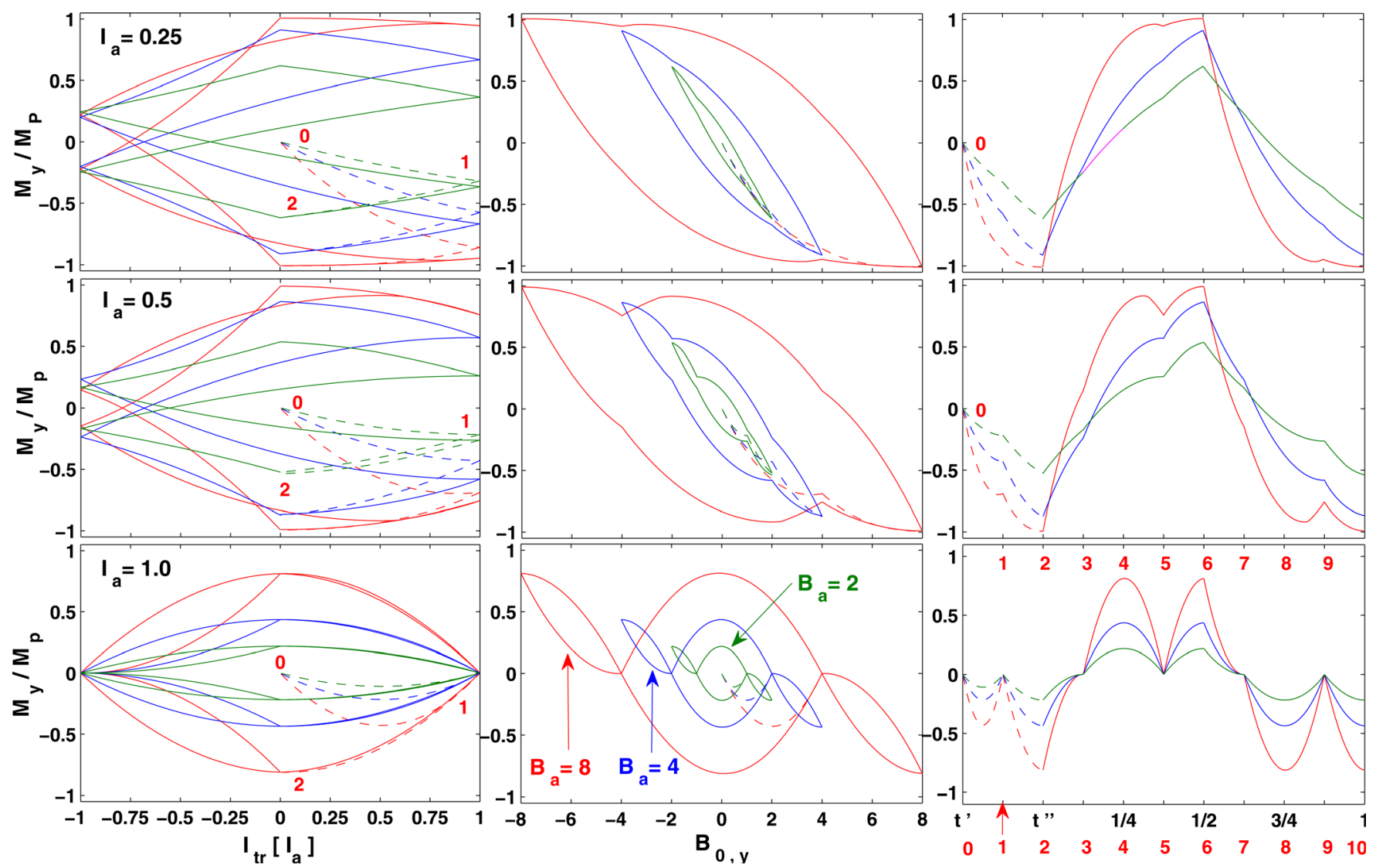

FIG. 11. The dimensionless magnetic moment $M_{y} / M_{p}$ for the ac asynchronous excitations displayed in Fig. 2(b) where the applied magnetic field has the role of dominant excitation. Curves are shown as function of the injected transport current $I_{t r}$ in units of their amplitude $I_{\mathrm{a}}$ (left column), the applied magnetic field $B_{0, y}$ (central column), or either by their temporal evolution (right column). Same color scheme to point out the amplitude of the ac magnetic field $\left(B_{\mathrm{a}}\right)$ has been used in all subplots.

frequency), which may counterbalance the local variation of the magnetic flux density produced by the other one in the zone of maximum heat release. Main features of the ac losses behavior by phase shifting between the electromagnetic sources can be easily explained through the above discussion, which is physically supported by assuming that the local density of magnetic flux $\mathbf{B}_{r_{i}}$ can be computed from the sum of the external field $\mathbf{B}_{0}$, and the density of magnetic flux produced by the induced screening currents of density $\mathbf{J}_{\mathbf{r}_{i}}$, i.e., $\mathbf{B}_{r_{i}}=\mathbf{B}_{0}\left(r_{i}\right)+\mathbf{B}_{\text {ind }}\left(\mathbf{r}_{i j}\right)$. Thus, at least for the $2 \mathrm{D}$ configurations studied along this paper, and after some mathematical manipulations, recalling the Ampere's law $\left(\nabla \times \mathbf{B}_{r_{i}}=\mu_{0} \mathbf{J}_{i}\right)$ and the intrinsic assumption for the magnetic vector potential $\mathbf{A}_{r_{i}}=\mathbf{A}_{0}\left(r_{i}\right)+\mathbf{A}_{\text {ind }}\left(\mathbf{r}_{i j}\right)$, the specific losses of power density $L \equiv \Delta L / \Delta t$ may be evaluated from the sum between the magnetization losses produced by variations of the external magnetic field

$$
\Delta L_{0}=\int_{\Omega} M_{y} d B_{0},
$$

and the inductive losses produced by the occurrence of screening currents (both, the magnetization currents and the injected current lines)

$$
\Delta L_{\text {ind }}=-\frac{\mu_{0}}{2} \int_{\Omega} \mathbf{B}_{r_{i}} \cdot \Delta \mathbf{B}_{r_{i}} d r
$$

such that $\Delta L=\Delta L_{0}+\Delta L_{\text {ind }}$. Here, we call reader's attention to the fact that for those cases where only the transverse magnetic field is applied, i.e., $I_{t r}(t)=0$, the flux front profile is defined by the optimal condition $B_{\text {ind }}(t)=-B_{0}(t)$, preceded of profiles accomplishing the material law $J= \pm J_{c}$, such that integration of Eq. (18) over the whole ac cycle becomes zero. However, when $I_{t r}(t) \neq 0$ the influence of Eq. (18) on the computation of the total ac losses is truly significant.

Recall that, the total ac loss decreases by ensuring minimal variation of $\Delta B_{r_{i}}$ along the whole sample. In fact, if the relative phase shift equals half a period, it means that the occurrence of the excitation peaks for the electromagnetic sources is likewise synchronous but is pointing in opposite directions. Thus, as the total ac loss may be calculated by integration of the excitations peak to peak, for this case $\Delta B_{r_{i}}$ is maximum and therefore also the actual ac loss. On the other hand, the forecast of the minimal variation of the set of integrands $\left(B_{r_{i}} \Delta B_{r_{i}}\right)$ may be done by considering a temporal displacement of a quarter of period [i.e., $\phi=\pi / 2$ for circular excitation functions], so that the local competition between the magnetic flux densities $\left|B_{0, i}\right|$ and $\left|B_{\text {ind }, i j}\right|$ minimizes as $B_{0, y}(\Omega)=0$ when $\left|I_{t r}\right|=I_{a}$. Then, under this simplified scenario, and at least for cases where the local distribution of screening currents is current-like (very low magnetic field, $b_{a} \approx 0$, and high transport current, $i_{a} \approx 1$ ), or field-like (high magnetic field, $b_{a} \gtrsim 1$, and very low transport 

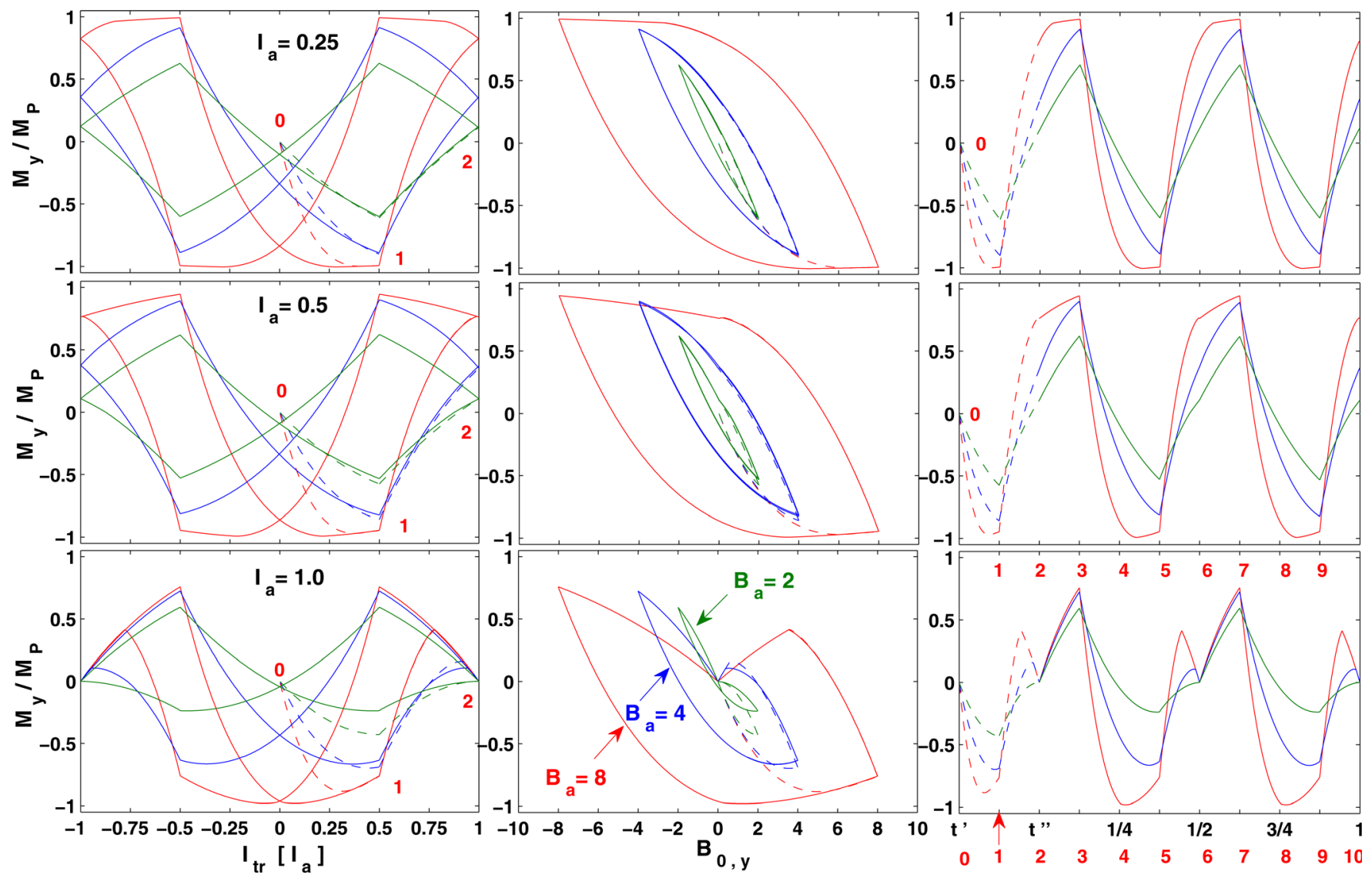

FIG. 12. The dimensionless magnetic moment $M_{y} / M_{p}$ for the ac asynchronous excitations displayed in Fig. 2(c) where the transport current has the role of dominant excitation. Curves are shown as function of the injected transport current $I_{t r}$ in units of their amplitude $I_{\mathrm{a}}$ (left column), the applied magnetic field $B_{0, y}$ (central column), or either by their temporal evolution (right column). Same color scheme to point out the amplitude of the ac magnetic field $\left(B_{\mathrm{a}}\right)$ has been used in all subplots.

current, $i_{a} \approx 0$ ), the minimal ac loss is envisioned to appear for a relative phase difference of a quarter of period. Likewise, the maximal ac loss may be predicted when both sources are fully synchronous or when there is a relative phase shift of half period. Latter facts agree with the analytical approaches for the slab ${ }^{60}$ and strip ${ }^{61}$ geometries. Also, in further agreement with the experimental evidences, ${ }^{43-45}$ within our statement we predict that as long as a phase shifting occurs, at least a minimal reduction of the ac loss should be observed.

Below, we concentrate on a different situation, occasionally found in practical devices, that will add new physical features to the ac loss scenario. In particular, we have considered the effect of introducing one of the excitations with an oscillating frequency twice as big as the other (see Fig. 2). Thus, calculation of the ac loss, i.e., integration of the local density of power dissipation $\mathbf{E} \cdot \mathbf{J}$ is made along the smaller frequency excitation. Surprisingly, the occurrence of double frequency effects can drastically alter the efficiency of the superconducting wires by increasing the ac losses, where we have found even greater values than those calculated for all the cases discussed above. In detail, hereinafter we will consider the following situations:

1. The injected transport current is the source within the double frequency regime [see Fig. 2(b)].
2. The temporal dynamics of the magnetic flux density associated with the external source of magnetic field shows a double frequency behavior [see Fig. 2(c)].

Most remarkable features for the flux dynamics, magnetic response, and ac losses for the above mentioned configurations are detailed below.

\section{B. Flux dynamics}

Before discussing the results obtained for the total ac loss in the set of experiments displayed in Fig. 2, some outstanding facts related to the rich phenomenology found for the local dynamics of the electromagnetic quantities are worth of mention. For instance, when the applied magnetic field and the transport current are synchronous, and their associated amplitudes $\left(B_{\mathrm{a}}, i_{\mathrm{a}}\right)$ are weak enough such that a flux-free core remains along the ac cycles (i.e., as long as $i_{\mathrm{a}}^{*} \neq 1$ and $b_{\mathrm{a}}^{*}<1$ ), it is more or less simple to identify the active zones in the ac cycles via the previous knowledge of the virgin branch (see Figs. 3 and 4). Therefore, explaining and obtaining the ac loss may be achieved if the distribution of screening currents is well known for the first half of the ac period. However, when the temporal dynamics of the isolated excitations shows an asynchronous response, this is not longer valid. The reason is that the hysteretic losses produced along the virgin branch are 

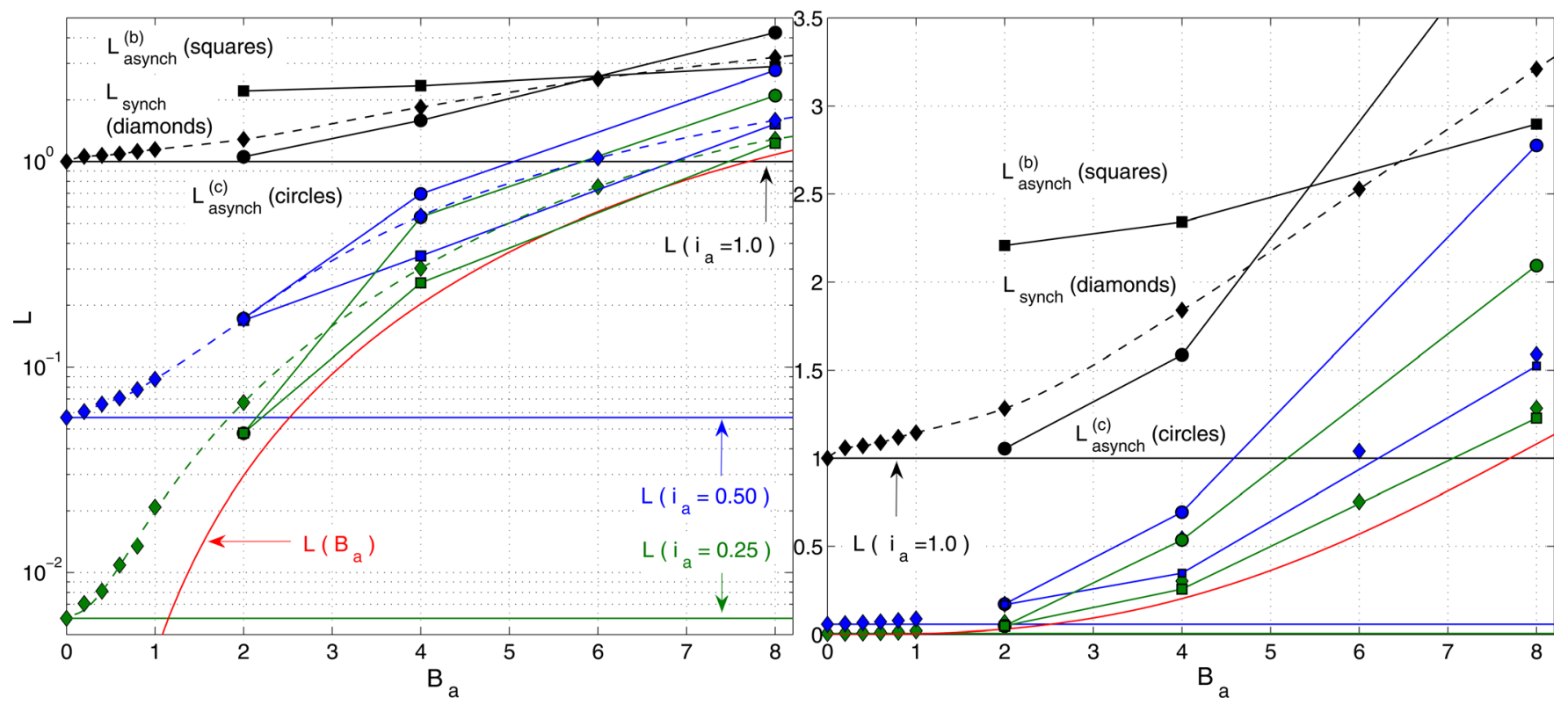

FIG. 13. Hysteretic ac losses per cycle for asynchronous sources accordingly to the excitations shown in Fig. 2(b) "Herein, $L_{\text {asynch }}^{(b)}$ : square-solid-lines," and Fig. 2(c) "Herein, $L_{\text {asynch }}^{(c)}$ : circle-solid-lines." The results are compared with the curve of losses for synchronous sources, $L_{\text {synch }} \equiv L\left(B_{\mathrm{a}}, I_{\mathrm{a}}\right)$ predicted above (Fig. 8), and the curves for isolated excitations $L\left(B_{\mathrm{a}}\right)$ and $L\left(I_{\mathrm{a}}\right)$. The whole set of results is also plotted in linear scale. Units for losses are $\left(\mu_{0} / 4 \pi\right) \omega R^{2} J_{c}^{2}$.

not monotonic concerning the temporal evolution of both electromagnetic excitations, such that the accruing hysteretic losses for the lower limit in the outer integral of Eq. (13) are different for the first and second half of the cyclic period. In other words, the distribution of screening currents in the first peak of the dominant excitation, i.e., that one with smaller frequency [time step 2 in Fig. 2] may drastically differ from those conceived in the second and third peaks [time steps 6 and 10]. Hence, the distribution of screening currents for the first half period of the ac cycle cannot be fetched through their distribution in the virgin branch, and therefore a proper description of the profiles of current density in an asynchronous ac regime must be done at least for the second half period of the dominant excitation (see Fig. 10).

We call reader's attention to the fact that a thorough follow-up of the local distribution of current density and

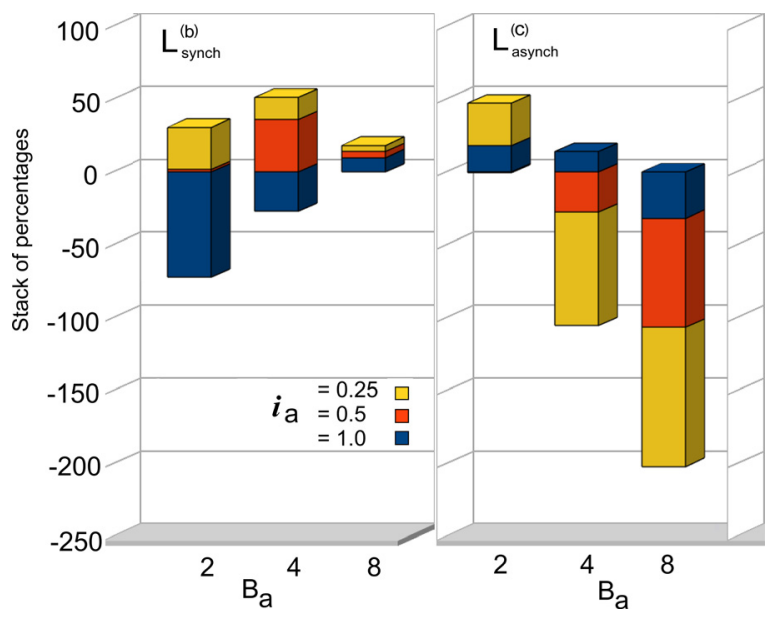

FIG. 14. Percent change between the ac loss for synchronous excitations, $L_{\text {synch }}$, and the losses $L_{\text {asynch }}^{(b)}$ (at the left-side) and $L_{\text {asynch }}^{(c)}$ (at the right-side), for combinations of three different amplitudes $B_{\mathrm{a}}$ and $i_{\mathrm{a}}$. lines of magnetic field for asynchronous sources with double frequency effects, including the virgin branch, can be found in the Fig. 13 of the supplementary material. Likewise, their corresponding profiles for the components of magnetic flux density are shown in Figs. 14 and 15, as well as the evolution of the local density of power dissipation $\mathbf{E} \cdot \mathbf{J}$ in Fig. $16 .^{57}$

Analyzing the distribution of current density profiles in Fig. 10 and their corresponding profiles for the local density of power dissipation $\mathbf{E} \cdot \mathbf{J}$, we found at least two interesting facts which are worth of mention. On the one hand, whether it is the magnetic field or the transport current, the excitation which leads the role of dominant, multiple domains or active zones may appear, which makes it tough to find out a feasible analytical solution for the flux front boundary in the high number of combinations between the amplitudes $B_{\mathrm{a}}$ and $i_{\mathrm{a}}$, specially if the pattern of current density is far away of the approaches for profiles of the kind current-like or field-like. On the other hand, a most striking fact revealed in Fig. 10 is that contrary to the behavior displayed for the local profiles of density of power dissipation $\mathbf{E} \cdot \mathbf{J}$, when both electromagnetic excitations are synchronous (see Fig. 5), in asynchronous cases the zone of heat release is no longer localized in one side of the superconducting sample. Thus, the idea of focusing heat release in some part of the superconductor requires a special attention in the synchronization of sources.

\section{Magnetic response}

Another interesting feature which derives from the study of asynchronous excitations is the actual possibility of finding "exotic" magnetization loops as a function of the ac sources (see Figs. 11 and 12), where the straightforward competition between the magnetization currents (by consumption) and the injected current lines may be visualized in terms of an average macroscopic measurement. 
In Fig. 11, the component of magnetic moment $M_{y}$ is displayed for the ac process displayed in Fig. 2(b), where the ac magnetic field dominates the cyclic period of excitation. As before, the whole set of results for $M_{y}$ has been renormalized according to the maximal expected value for the magnetic moment when only applied magnetic field is considered ( $M_{p}=2 / 3$ in dimensionless units). Thus, curves are shown as function of the isolated electromagnetic excitations, $I_{t r}$ (in units of their associated ac amplitude $I_{\mathrm{a}}$ ) at the left column, $B_{0, y}$ at the central column, as well as by the time defining the first steady-period (right column). Notice that the virgin branch which does not play any role for the integration of the ac losses per cyclic periods is shown through dashed lines. Furthermore, results have been organized accordingly to the associated amplitudes for the applied density of magnetic flux $B_{\mathrm{a}}$, such that $B_{\mathrm{a}}=2$ corresponds to the green curves, $B_{\mathrm{a}}=4$ to the blue curves, and $B_{\mathrm{a}}=8$ to the red curves. Likewise, the set of curves shown for each row can be straightforwardly associated with a single value for the amplitude of the ac transport current, $i_{\mathrm{a}}=0.25$ (first row), $i_{\mathrm{a}}=0.5$ (second row), and $i_{\mathrm{a}}=1$ (third row). Analogously, the corresponding set of curves obtained for the component of magnetic moment $M_{y}$ in those cases where the ac transport current dominates the cyclic period of excitation is shown in the same fashion above described in Fig. 12.

Outstandingly, whether $B_{0, y}$ (Fig. 11) or $I_{t r}$ (Fig. 12) is the dominant excitation, and for low values of $I_{\mathrm{a}}$, the magnetization loops as function the magnetic flux density $M_{y}\left(B_{0, y}\right)$ show a Bean-like behavior. As the value of $i_{\mathrm{a}}$ increases, notorious deformations of the Bean-like structures for the magnetic moment appear. Nevertheless, the behavior is radically different comparing the double frequency effects provided by one or another excitation, as it is explained below.

\section{Transport current with double frequency}

On the one hand, when it is the ac transport current, $I_{t r}$, that shows a double frequency, the magnetization curves $M_{y}\left(B_{0, y}\right)$ display a symmetric behavior in the regions (leftright) of the periods $\left[B_{\mathrm{a}} \rightarrow-B_{\mathrm{a}}\right]$ and $\left[-B_{\mathrm{a}} \rightarrow B_{\mathrm{a}}\right]$ (see Fig. 11). On the contrary, for $M_{y}\left(I_{t r}\right)$, one can notice the existence of a symmetrization of the curves of magnetic moment regarding their positive and negative values (up/ down). Evidently, the steady-states where the maximum consumption of the magnetization currents occurs, always arise when the asynchronous ac excitation $\left[B_{0, y}, I_{t r}\right]$ reaches the values for the current's peaks [see Fig. 2(b)], i.e., for the time-steps (3) $\left[B_{\mathrm{a}} / 2,-I_{\mathrm{a}}\right],(5)\left[-B_{\mathrm{a}} / 2, I_{\mathrm{a}}\right],(7)\left[-B_{\mathrm{a}} / 2,-I_{\mathrm{a}}\right]$, and (9) $\left[B_{\mathrm{a}} / 2, I_{\mathrm{a}}\right]$. Then, by increasing (decreasing) the value of $I_{\mathrm{a}}$, a progressive decreasing (increasing) of the magnetic moment at these points ends up in the simultaneous collapsing of the magnetization curves $\left(M_{y} \equiv 0\right)$ for the half periods of the dominant excitation $B_{0, y}$, as long as the transport current reaches the threshold value of critical current, i.e., $i_{\mathrm{a}}= \pm 1$. Latter fact is followed by the symmetrization of the loops, either as functions of $B_{0, y}$ and $I_{t r}$, into characteristic lenticular shapes bounded by two non-connected magnetization curves, both defined by the elapsed periods in which the time derivative of $I_{t r}(t)$ is positive, i.e., for the temporal branches $(3 \Rightarrow 5)$ and $(7 \Rightarrow 9)$. Then, the connecting curves for the abovementioned magnetization branches show characteristic lashing shapes when the time derivative of $I_{t r}(t)$ is negative. Remarkably, as a consequence of this process, the output signal $M_{y}(t)$ does not show the low-pass filtering effects conceived for synchronous excitations.

\section{Applied magnetic field with double frequency}

On the other hand, when it is the ac density of magnetic flux, $B_{0, y}$, the electromagnetic source disclosing the double frequency effect [Fig. 2(c)], the calculated curves of magnetization $M_{y}\left(B_{0, y}\right)$ are outstandingly different. In fact, there are no symmetry conditions which may be observed in this representation (2nd column in Fig. 12). However, for the set of magnetization curves as a function of the transport current, $M_{y}\left(I_{t r}\right)$, we have noticed a well-defined symmetrization of the magnetization loops regarding to the positive and negative values of $I_{t r}(t)$ (left/right). Notwithstanding, in terms of the magnetization curves $M_{y}\left(B_{y}\right)$, there is also a further fact to be mentioned. Strikingly, by using this representation it is possible to note that the steady-states where the consumption of the magnetization currents becomes evident are mainly present along the period in which the time derivative of $B_{0, y}(t)$ is negative, whilst the ac transport current evolves through the current's peaks, i.e., for the temporal branches defined by the time-steps (1) $\left[B_{\mathrm{a}}, I_{\mathrm{a}} / 2\right]$ to $(3)\left[-B_{\mathrm{a}}, I_{\mathrm{a}} / 2\right]$, and (5) $\left[B_{\mathrm{a}},-I_{\mathrm{a}} / 2\right]$ to (7) $\left[-B_{\mathrm{a}},-I_{\mathrm{a}} / 2\right]$. Moreover, the magnetic response of the superconductor is not monotonic along these branches. For example, from the time step (5) until the time step (6) $\left[0,-I_{\mathrm{a}}\right]$, the electromagnetic excitations, $B_{0, y}$ and $I_{t r}$, have the same tendency (both decreasing). However, if $I_{\mathrm{a}}$ tends to the value of the critical current, $M_{y}$ may increase and decrease within the same period. Then, from the time step (6) to the time step (7) both evolve in opposite directions, but however the magnetic moment always increases along this period. On the other hand, for the following analogous branch in the ac period of magnetic field, say the timesteps (9) to (11), the magnetic moment curve is the same, but the competition between the electromagnetic sources $B_{0, y}$ and $I_{t r}$ is opposite to the aforementioned evolution. Thus, depending on the intensities of the electromagnetic sources, both reduction and enhancement of the hysteretic ac losses may be envisaged when there is a difference between the oscillating excitation frequencies. This is in contrast to the monotonic behavior for synchronous sources.

\section{AC losses in asynchronous systems}

In Fig. 13, the hysteretic ac losses calculated for the experimental configurations conceived in Fig. 2 [panes (b) and (c)] are shown. To be specific, $L_{\text {asynch }}^{(b)}$ and $L_{\text {asynch }}^{(c)}$ are shown in terms of the amplitude of the applied density of magnetic flux $B_{\mathrm{a}}$, whilst the different values for $i_{\mathrm{a}}$ are pointed in terms of the sequence of colors for the dc loss curves depicted in Fig. 8. Likewise, results are compared with the corresponding curves for the actual ac loss when the synchronous electromagnetic excitations were considered, i.e., $L_{\text {synch }} \equiv L\left(B_{\mathrm{a}}, i_{\mathrm{a}}\right)$. Outstandingly, for both cases, 
remarkable variations of the ac loss occur. Thus, with the aim of providing a clearest understanding of the range of variations in the ac loss curve for the configurations abovementioned, and further help the readers in the visualization of the numerical data, in Fig. 14 we show the percentage relation between the calculated losses for synchronous excitations, $L_{\text {synch }}$, and the calculated losses for the asynchronous cases, $L_{\text {asynch }}^{(b)}$ and $L_{\text {asynch }}^{(c)}$.

It is worth noticing that when the applied magnetic field provides the dominant oscillating period (the impressed ac transport current shows a relative double frequency, Fig. 2(b)), the resulting comparison between the calculated losses $L_{\text {asynch }}^{(b)}$ and $L_{\text {synch }}$ for high values of $B_{\text {a }}$ [Fig. 14] shows a small but sizable increase of the hysteretic loss as a consequence of the double frequency effect $(\sim 4 \%-10 \%$ for $B_{\mathrm{a}} \equiv B_{p}=8$ ). Then, assuming that $i_{\mathrm{a}} \equiv 1$, we have found that the ac loss reduces as $B_{\mathrm{a}}$ decreases. However, an outstanding fact is that for the lowest values of $B_{\mathrm{a}}$ and $i_{\mathrm{a}}$, a notorious increase of the actual ac losses appears. For example, for $B_{\mathrm{a}}=B_{p} / 2=4$ and $i_{\mathrm{a}}=0.5$, deviation is about $36 \%$. Likewise, for $B_{\mathrm{a}}=B_{p} / 4=2$ and $i_{\mathrm{a}}=0.25$, deviation is about $29 \%$.

On the other hand, when the electromagnetic source with the double frequency is the applied magnetic field [Fig. 2(c)], the resulting ac loss $\left(L_{\text {asynch }}^{(c)}\right)$ for high values of $B_{\text {a }}$ shows a significant reduction as compared to the predicted losses for synchronous configurations ( $\sim 95 \%$ for $B_{\mathrm{a}} \equiv B_{p}=8$ and $i_{\mathrm{a}}=0.25$ ). However, by reducing $B_{\mathrm{a}}$ a notorious increase of the ac loss may be revealed depending on the value of $i_{\mathrm{a}}$. In fact, we call readers' attention about the relative increase of the ac losses as compared to those of the synchronous cases: $\sim 14 \%$ for $\left(B_{\mathrm{a}}=4, i_{\mathrm{a}}=1\right)$, and $\sim 18 \%$ for $\left(B_{\mathrm{a}}=2, i_{\mathrm{a}}=1\right)$. Moreover, for those cases with the lower values of $B_{\mathrm{a}}$, i.e., $B_{\mathrm{a}}=2$, increases of the ac loss are also found for $i_{\mathrm{a}}=0.25$ ( $\sim 29 \%)$, whilst for the intermediate case $\left[B_{\mathrm{a}}=2, i_{\mathrm{a}}=0.5\right]$ it shows a reduction of the ac loss of less than $1 \%$. The remarkable point here is that for asynchronous excitations of $B_{0}$ and $I_{t r}$, reductions of the ac losses can be only asserted if both sources evolve with the same frequency, i.e., if one is restricted to shifts in phase.

\section{CONCLUDING REMARKS}

In this paper, we have presented a thorough study of the local and global electromagnetic response of a straight, infinite, cylindrical type-II superconducting wire subject to diverse ac-configurations of transverse magnetic flux density $B_{0}(t)$ and/or longitudinal transport current flow $I_{t r}(t)$. We have assumed that the superconductor follows the celebrated critical state model with a constant threshold for the critical current density $J_{c}$, such that $\left|I_{t r}\right| \leq\left|J_{c}\right| \pi R^{2}$. The problem is posed over a mesh of virtual filamentary wires each carrying a current $I_{i}$ across a surface $s_{i}$, filling up the whole cross section of the superconducting wire whose area is defined by $\Omega=\pi R^{2}$.

After a brief theoretical review that concentrates on the physical nature of our variational approach (Sec. II), we have performed extensive numerical calculations for several amplitudes of the impressed transport current, $I_{\mathrm{a}}$, as well as the amplitude of the magnetic flux density associated with the external excitation source, $B_{\mathrm{a}}$, for two different regimes of excitation. On the one hand, in Sec. III we have studied the electromagnetic response of superconducting wires subjected to synchronous excitations, particularly when the wire is in one of the two possible scenarios: (1) the superconducting wire has not been exposed earlier to any kind of electromagnetic excitation such that it is in its virgin state (Fig. 1), or (2) the superconducting wire has been previously magnetized (Fig. 7). On the other hand, Sec. IV has addressed a study about the effects of considering different asynchronous regimes for the oscillating electromagnetic sources, with attention to cumbersome scenarios such as electromagnetic sources with double frequency effects (Fig. 2). Thus, for each of the above cases, and in order to understand the influence of the electromagnetic excitations involved in the macroscopical physical processes found in this kind of systems, we have presented a detailed study of the local dynamics of the distribution of screening currents, $I_{i}= \pm J_{c} s_{i}$ or 0 , as well as the related local density of power dissipation $\mathbf{E} \cdot \mathbf{J}$ along a cyclic oscillating period. Likewise, for a wide number of experiments, we have presented the full behavior of the magnetic flux density vector $\mathbf{B}$ in supplementary material. ${ }^{57}$ For a closer connection with the most common experimental observables, we have calculated the wire's magnetic moment $\mathbf{M}$ and the hysteretic losses $L$ as a function of the ac external excitations, and their comparison with classical analytical approaches has been featured. Our main conclusions concerning the underlying physics of the synchronous and asynchronous regimes are detailed below.

\section{A. SC wires subjected to synchronous excitations}

(1) The local distribution of screening currents when the simultaneous action of the electromagnetic excitations $B_{0, y}$ and $I_{t r}$ is conceived, may be described as the consumption of the magnetization currents (screening currents induced by the external magnetic field) by effect of the occurrence of injected current lines (constrained by the condition $\sum_{i} I_{i}=I_{t r}$ ). As a result of this, the flux front profile is displaced from the geometrical center of the wire towards one of the sides by a kind of "Lorentz force" effect on the injected current lines (see, e.g., Figs. 3 and 4).

(2) For low magnetic fields $\left(B_{\mathrm{a}} \leq 2\right.$, in units of $\left.\mu_{0} J_{c} R / 4 \pi\right)$, the distribution of screening currents is current-like as long as the peak intensity of the transport current is high $\left(i_{\mathrm{a}} \geq 0.5\right.$, in units of the critical current) that the "magnetization lines" in counter direction to the injected current lines may be neglected (Fig. 3). Likewise, for higher values of $B_{\mathrm{a}}$ and lower values of $I_{\mathrm{a}}$, field-like profiles may be envisaged (Fig. 4). Nevertheless, for most of the possible combinations between $B_{\mathrm{a}}$ and $I_{\mathrm{a}}$, nonsymmetric distributions of the screening currents in a nothing-like fashion have been predicted (Figs. 1-7 in supplementary material ${ }^{57}$ ).

(3) Remarkable distortions of the magnetic flux density outside of the superconducting wire are mainly observed at 
the instants when the synchronous excitation tends to zero in the ac excitation. On the other hand, the maximal density of magnetic flux occurs in the side opposite to the location of the flux free core. Thus, recalling Eq. (18), the strongest localization of the density of magnetic flux in one side of the active zone of the material produces a remarkable localization of the local hysteretic losses in such manner that the heat release from the superconducting wire is highly localized too (Fig. 5).

(4) Regarding the magnetic moment curves (Fig. 6), we argue that only for small values of $I_{\mathrm{a}}$, Bean-like loops are expected. However, as $I_{\mathrm{a}}$ increases, the derived effect by the consumption of the magnetization currents is most prominent, ending up with the symmetrization of the magnetization loop for cyclic periods as function of $B_{0}$ or $I_{t r}$ into striking lenticular shapes. As a consequence of this process, a distinct low-pass filtering effect comes to the fore which, in the case of the triangular input excitations with $I_{\mathrm{a}}=I_{c}$, yields a nearly perfect sinusoidal (first-harmonic) output signal $M_{y}(t)$.

(5) If the superconducting wire has been magnetized before switching on the synchronous ac excitation $\left(\mathbf{B}_{0}, I_{t r}\right)$, say at $t=t^{\prime}$ (Fig. 7), the center of the magnetization loop drifts from $\mathbf{M}(0,0)$ towards $\mathbf{M}\left(B_{0}\left(t^{\prime}\right), 0\right)$, such that the corners of the magnetization loop $\mathbf{M}\left( \pm B_{\mathrm{a}}, \pm I_{\mathrm{a}}\right)$ lie on the excitation coordinates $\left(B_{0}\left(t^{\prime}\right) \pm B_{\mathrm{a}}, \pm I_{\mathrm{a}}\right)$. The area enclosed by the magnetization loop remains the same, and so does the power. Nevertheless, in these cases, the profiles drawn for the screening currents have revealed highly intricate patterns regarding to the coexistence of the magnetization currents and the injected current lines, as well as defining the flux front profile (Fig. 7 in supplementary material ${ }^{57}$ ).

(6) Our calculation of the actual hysteretic losses by means the general definition $\delta L=\int_{v o l} \mathbf{E} \cdot \mathbf{J}$, reveals important differences concerning the approximate formulae customarily used. In fact, we have shown that the actual ac losses are always higher than those envisaged by the linear superposition of contributions due to either type of ac excitation (Fig. 8). Comparisons reveal the important fact that the linear approach $L\left(B_{\mathrm{a}}\right)+L\left(i_{\mathrm{a}}\right)$ is only appropriate for high strengths of the magnetic flux density and low transport currents (screening currents with distribution field-like), as well as for low magnetic field and high transport current (screening currents with distribution current like). Likewise, for high amplitudes of the magnetic flux density $\left(B_{\mathrm{a}} \geq B_{p}\right)$ and for any value of the transport current, significant reductions of the actual hysteretic loss may be envisaged if one compares it with the predicted losses for a wire carrying a dc current instead to the ac case (Fig. 9).

\section{B. SC wires subjected to asynchronous excitations}

Our analysis of the underlying physics behind the local and global electromagnetic response of superconducting wires subjected to the simultaneous action of electromagnetic excitations $B_{0}(t)$ and $I_{t r}(t)$ in synchronous oscillating regimes has revealed that the ac loss may be controlled by locally reducing the total magnetic flux density resulting from the addition of the external magnetic field and that induced by the concomitant occurrence of magnetization currents and the injected current lines. Thus, either by displacing in time the electromagnetic excitations (phase shift), by introducing changes in frequency, or simply by considering excitation branches with time derivatives in counter directions, one can help to counterbalance the local density of magnetic flux in the zone of maximum heat release, which is further translated to the reduction of the hysteretic losses.

As long as both excitations evolve with the same oscillating frequency, it is possible to assert that at least a minimal reduction of the ac loss should appear for relative phase changes between the electromagnetic excitations. This is in agreement with the experimental and numerical evidences reported in Refs. 43-45. For example, by the simple overlapping between the excitation curves of the electromagnetic sources, it is evident that the maximal losses are envisaged for the synchronous cases, as well as for those cases with a relative change in phase of half excitation period. Likewise, minimal losses are attained around a phase shifting of a quarter of period, although its exact position as a function of the electromagnetic excitations straightforwardly depends on the entangled competition between the external magnetic field and, the induced fields by the injected current lines and the so called magnetization currents. Thus, unless the distribution of screening currents show patterns of the kind current-like or field-like is not possible assert that the lower ac loss is given for a change of phase of a quarter of period.

Within the above scenario, we have argued that predictions are not straightforward for non-trivial time dependencies of the simultaneous electromagnetic excitations. Along this line, we have introduced the consideration of the socalled double frequency effects, which arise when one of the electromagnetic sources, $B_{0}(t)$ or $I_{t r}(t)$, shows a double oscillating frequency (Fig. 2). The most outstanding observations about the local and global behavior of the involved electromagnetic quantities are as follows:

(1) For asynchronous excitations, the distribution of screening currents in the first peak of the dominant excitation (i.e., that with a longer period) may be strongly different as compared to the attained distributions for the subsequent excitation peaks (Fig. 13 in supplementary material $^{57}$ ). Thus, the first peak of excitation cannot be considered as a steady-state for integrating the ac loss when the integral is reduced to half period of excitation. In other words, the knowledge of the distribution of screening currents in the time elapsed for the second half-period of the dominant excitation is relevant. The latter fact has been experimentally recognized in Ref. 43.

(2) Whatever electromagnetic excitation carries the double frequency, we have found that, in the ac regime, complex arrays of domains connected by boundary lines with currents switching between $I_{c}$ and $-I_{c}$ appear. Furthermore, contrary to the strong localization of the local density of power losses observed in cases with synchronous excitations, for asynchronous excitations the active zone with 
higher heat release is no longer focused at only one side of the superconducting wire (Fig. 10).

(3) As far as concerns to the magnetic response of the superconducting wire when double frequency effects are incorporated, exotic magnetization loops are predicted (see Figs. 11 and 12). Outstandingly, for low amplitudes of the transport current, $I_{\mathrm{a}}$, Bean-like loops may be observed for any of the above mentioned cases. However, as $I_{\mathrm{a}}$ increases, strongest differences between the magnetization loops arise, and the global behavior is radically different to the synchronous cases.

(4) A comprehensive analysis of the magnetization curves as a function of the temporal evolution of the electromagnetic excitations has been carried out. On the one hand, several symmetry conditions for the magnetization loop, when the electromagnetic excitation with double frequency is either $I_{t r}$ or $B_{0}$, concerning to the amplitudes $I_{\mathrm{a}}$ and $B_{\mathrm{a}}$ have been illustrated. On the other hand, we have shown that in order to attain the low-pass filtering effect in the temporal evolution of the magnetization curve when $I_{\mathrm{a}} \rightarrow I_{c}$, it becomes absolutely necessary to assure that both oscillating excitations evolve synchronous in time.

(5) Accordingly to the different setups in panes (b) and (c) of Fig. 2, we have calculated the hysteretic ac loss for several values of $B_{\mathrm{a}}$ and $I_{\mathrm{a}}$, when the dominant excitation is either the applied magnetic field, $L_{a s y n c h}^{(b)}$, or the impressed transport current, $L_{\text {asynch }}^{(c)}$ (Fig. 13). By comparing them with the hysteretic ac loss predicted for the synchronous cases (see also Fig. 14), we have shown that the ac loss may either increase or decrease by double frequency effects when asynchronous excitations are involved. The relative intensities of the excitations $B_{\mathrm{a}}$ and $I_{\mathrm{a}}$ play an additional role on this. Then, the big number of possible combinations makes it imperative to have a previous knowledge of the operational environment of the superconducting wire, for attaining valid predictions of the actual ac losses.

\section{ACKNOWLEDGMENTS}

This work was supported by the Spanish MINECO and the FEDER program (MAT2011-22719 and ENE201129741 projects), DGA Grant T12/2011. Funding provided by the Spanish CSIC JAE program is gratefully acknowledged. H. S. Ruiz thanks Y. A. Genenko and S. V. Yampolskii from the Technische Universität Darmstadt (Fachgebiet Materialmodellierung) for helpful discussions.

${ }^{1}$ C. P. Bean, Rev. Mod. Phys. 36, 31 (1964); J. Appl. Phys. 41, 2482 (1970).

${ }^{2}$ H. S. Ruiz, A. Badía-Majós, Y. A. Genenko, H. Rauh, and S. V. Yampolskii, Appl. Phys. Lett. 100, 112602 (2012).

${ }^{3}$ W. J. Carr, Jr., M. S. Walker, and J. H. Murphy, J. Appl. Phys. 46, 4048 (1975).

${ }^{4}$ V. B. Zenkevitch, V. V. Zeltov, and A. S. Romanyuk, Cryogenics 18, 93 (1978); Sov. Phys. Dokl. 25, 210 (1980); V. B. Zenkevitch, A. S. Romanyuk, and V. V. Zeltov, Cryogenics 20, 703 (1980).

5 J. V. Minervini, IEEE Trans. Magn. 25, 2113 (1989).
${ }^{6}$ W. J. Carr, AC Loss and Macroscopic Theory of Superconductors, 2nd ed. (Taylor \& Francis, New York, 2001).

${ }^{7}$ A. V. Gurevich, R. G. Mints, and A. L. Rakhmanov, Physics of Composite Superconductors (Nauka Publishers, 1987) [Engl. translation: Begell House, New York, 1997] (in Russian).

${ }^{8}$ G. W. Swan, J. Math. Phys. 9, 1308 (1968).

${ }^{9}$ M. R. Halse, J. Phys. D 3, 717 (1970).

${ }^{10}$ W. T. Norris, J. Phys. D 3, 489 (1970).

${ }^{11}$ E. H. Brandt and M. Indenbom, Phys. Rev. B 48, 12893 (1993); E. H. Brandt, M. V. Indenbom, and A. Forkl, Europhys. Lett. 22, 735 (1993).

${ }^{12}$ E. Zeldov, J. R. Clem, M. McElfresh, and M. Darwin, Phys. Rev. B 49, 9802 (1994).

${ }^{13}$ Y. Mawatari, Phys. Rev. B 83, 134512 (2011).

${ }^{14}$ M. Ashkin, J. Appl. Phys. 50, 7060 (1979); M. Ashkin and G. R. Wagner, J. Appl. Phys. 60, 2477 (1986).

${ }^{15}$ C. Y. Pang, A. M. Campbell, and P. G. McLauren, IEEE Trans. Magn. 17, 134 (1981).

${ }^{16}$ K. L. Telschow and L. S. Koo, Phys. Rev. B 50, 6923 (1994).

${ }^{17}$ Y. E. Kuzovlev, JETP Lett. 61, 1000 (1995).

${ }^{18}$ K. V. Bhagwat and D. Karmakar, Pramana 57, 763 (2001); D. Karmakar and K. V. Bhagwat, Physica C 398, 20 (2003).

${ }^{19}$ F. Gömöry, R. Tebano, A. Sanchez, E. Pardo, C. Navau, I. Husek, F. Strycek, and P. Kovac, Supercond. Sci. Technol. 15, 1311 (2002).

${ }^{20}$ B. ten Haken, J. J. Rabbers, and H. H. J. ten Kate, Physica C 377, 156 (2002).

${ }^{21}$ R. Navarro and L. J. Campbell, Phys. Rev. B 44, 10146 (1991).

${ }^{22}$ N. Amemiya and Y. Ohta, Physica C 357-360, 1134 (2001).

${ }^{23}$ N. Nibbio and S. Stavrev, IEEE Trans. Appl. Supercond. 11, 2627 (2001).

${ }^{24}$ S. Stavrev, B. Dutoit, and N. Nibbio, IEEE Trans. Appl. Supercond. 12, 1857 (2002).

${ }^{25}$ Z. Hong, L. Ye, M. Majoros, and A. M. Campbell, J. Supercond. Novel Magn. 21, 205 (2008).

${ }^{26}$ L. Prigozhin, Physica D 197, 197 (2004).

${ }^{27}$ J. W. Barrett and L. Prigozhin, Math. Models Meth. Appl. Sci. 20, 679 (2010).

${ }^{28}$ A. M. Campbell, Supercond. Sci. Technol. 20, 292 (2007); A. M. Wolsky and A. M. Campbell, Supercond. Sci. Technol. 21, 075021 (2008).

${ }^{29}$ A. Badía-Majós, C. López, and H. S. Ruiz, Phys. Rev. B 80, 144509 (2009); and references therein.

${ }^{30}$ H. S. Ruiz and A. Badía-Majós, Supercond. Sci. Technol. 23, 105007 (2010).

${ }^{31}$ R. Hancox, Proc. IEEE 113, 1221 (1966).

${ }^{32}$ W. J. Carr, IEEE Trans. Magn. 15, 240 (1979).

${ }^{33}$ N. Schönborg, J. Appl. Phys. 90, 2930 (2001).

${ }^{34}$ N. Amemiya, K. Miyamoto, S. Murasawa, H. Mukai, and K. Ohmatsu, Physica C 310, 30 (1998).

${ }^{35}$ T. Yazawa, J. J. Rabbers, B. ten Haken, H. H. J. ten Kate, and Y. Yamada, Physica C 310, 36 (1998).

${ }^{36}$ T. Yazawa, J. J. Rabbers, O. A. Shevchenko, B. ten Haken, and H. H. J. ten Kate, IEEE Trans. Appl. Supercond. 9, 797 (1999).

${ }^{37}$ S. Zannella, L. Montelatici, G. Grenci, M. Pojer, L. Jansak, M. Majoros, G. Coletta, R. Mele, R. Tebano, and F. Zanovello, IEEE Trans. Appl. Supercond. 11, 2441 (2001).

${ }^{38}$ R. Tebano, R. Mele, V. Boffa, F. Gömöry, F. Strýček, and E. Seiler, Int. J. Mod. Phys. B 17, 528 (2003).

${ }^{39}$ H. Tonsho, S. Fukui, T. Sato, M. Yamaguchi, S. Torii, T. Takao, and K. Ueda, IEEE Trans. Appl. Supercond. 13, 2368 (2003).

${ }^{40}$ J. Ogawa, M. Shiokawa, M. Ciszek, and O. Tsukamoto, IEEE Trans. Appl. Supercond. 13, 1735 (2003).

${ }^{41}$ N. Enomoto and N. Amemiya, Physica C 412-414, 1050 (2004).

${ }^{42}$ S. Stavrev, F. Grilli, B. Dutoit, and S. P. Ashworth, Supercond. Sci. Technol. 18, 1300 (2005).

${ }^{43}$ D. N. Nguyen, S. V. Pamidi, D. C. Knoll, G. Zhang, and J. Schwartz, J. Appl. Phys. 98, 073902 (2005).

${ }^{44}$ D. N. Nguyen, P. V. P. S. S. Sastry, G. M. Zhang, D. C. Knoll, and J. Schwartz, IEEE Trans. Appl. Supercond. 15, 2831 (2005).

${ }^{45}$ M. Vojenčiak, J. Šouc, J. M. Ceballos, F. Gömöry, B. Klinčok, E. Pardo, and F. Grilli, Supercond. Sci. Technol. 19, 397 (2006).

${ }^{46}$ E. Pardo, F. Gömöry, J. Šouc, and J. M. Ceballos, Supercond. Sci. Technol. 20, 351 (2007).

${ }^{47}$ E. Pardo, A. Sanchez, D.-X. Chen, and C. Navau, Phys. Rev. B 71, 134517 (2005).

${ }^{48}$ E. Pardo, D.-X. Chen, A. Sanchez, and C. Navau, Supercond. Sci. Technol. 17, 83 (2004). 
${ }^{49}$ E. Pardo, A. Sanchez, and C. Navau, Phys. Rev. B 67, 104517 (2003).

${ }^{50}$ W. Pi, Y.-S. Wang, J. Dong, and L. Chen, Chin. Phys. Lett. 27, 037401 (2010).

${ }^{51}$ K. P. Thakur, A. Raj, E. H. Brandt, J. Kvitkovic, and P. V. P. S. S. Sastry, Supercond. Sci. Technol. 24, 065024 (2011).

${ }^{52}$ K. P. Thakur, A. Raj, E. H. Brandt, and S. V. Pamidi, Supercond. Sci. Technol. 24, 045006 (2011).

${ }^{53}$ Y. Iwasa, Case Studies in Superconducting Magnets: Design and Operational Issues (Springer Science+Business Media, New York, 2009).

${ }^{54} \mathrm{P}$. Wanderer, See http://www.bnl.gov/magnets/magnet_files/Publications/ BNL-75350-2006-CP.pdf for "Exotic magnets for accelerators," in 19th International Conference on Magnet Technology, Superconducting Magnet Division, Brookhaven National Laboratory.

${ }^{55}$ V. S. Kashikhin, N. Andreev, V. V. Kashikhin, I. Novitski, and A. Zlobin, IEEE Trans. Appl. Supercond. 16, 1274 (2006).
${ }^{56}$ P. He, F. T. Wang, Z. M. Chen, W. G. Chen, Z. Y. Chen, J. W. Zhu, Y. Ren, and J. J. Li, IEEE Trans. Appl. Supercond. 20, 2331 (2010).

${ }^{57}$ See supplementary material at http://dx.doi.org/10.1063/1.4804931 for a complete visualization of the time-dependent local electromagnetic properties of the type-II superconducting wire and the different regimes considered along this paper.

${ }^{58}$ H. S. Ruiz, A. Badía-Majós, Y. A. Genenko, and S. V. Yampolskii, IEEE Trans. Appl. Supercond. 23, 8000404 (2013).

${ }^{59}$ V. B. Zenkevitch, V. V. Zheltov, and A. S. Romanyuk, Hysteretic Losses in Windings Made up Conductores Comprising Round Superconducting Filaments (Institute of High Temperatures of the USSR, Academy of Sciences, Moscow, 1983).

${ }^{60}$ S. Takács, Supercond. Sci. Technol. 20, 1093 (2007).

${ }^{61}$ Y. Mawatari and K. Kajikawa, Appl. Phys. Lett. 90, 022506 (2007). 